CRYSTALLOGRAPHIC COMMUNICATIONS

ISSN 2056-9890

Received 19 September 2019

Accepted 29 October 2019

Edited by J. Ellena, Universidade de Sâo Paulo, Brazil

Keywords: crystal structure; Ni metal-organic coordination network; terephthalate; layered structure.

CCDC reference: 1962320

Supporting information: this article has supporting information at journals.iucr.org/e

\section{Crystal structure and Hirshfeld surface analysis of poly[tris $\left(\mu_{4}\right.$-benzene-1,4-dicarboxylato)tetrakis(di- methylformamide)trinickel(II)]: a two-dimensional coordination network}

\author{
Cesario Ajpi, ${ }^{\text {a }}$ Leopoldo Suescun, ${ }^{b_{*}}$ Naviana Leiva, ${ }^{\text {a }}$ Anders Lundblad, ${ }^{c}$ Göran \\ Lindbergh $^{\mathrm{d}}$ and Saul Cabrera ${ }^{\mathrm{a} *}$
}

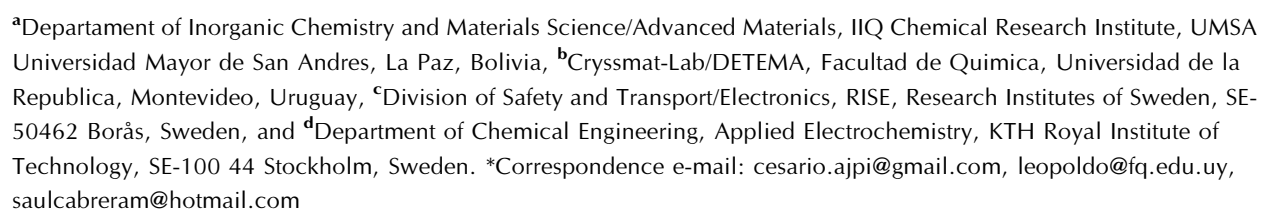

The crystal structure of the title compound, $\left[\mathrm{Ni}_{3}\left(\mathrm{C}_{8} \mathrm{H}_{4} \mathrm{O}_{4}\right)_{3}\left(\mathrm{C}_{3} \mathrm{H}_{7} \mathrm{NO}\right)_{4}\right]$, is a twodimensional coordination network formed by trinuclear linear $\mathrm{Ni}_{3}(\mathrm{tp})_{3}(\mathrm{DMF})_{4}$ units $(\mathrm{tp}=$ terephthalate $=$ benzene-1,4-dicarboxylate and DMF $=$ dimethylformamide) displaying a characteristic coordination mode of acetate groups in polynuclear metal-organic compounds. Individual trinuclear units are connected through tp anions in a triangular network that forms layers. One of the DMF ligands points outwards and provides interactions with equivalent planes above and below, leaving the second ligand in a structural void much larger than the DMF molecule, which shows positional disorder. Parallel planes are connected mainly through weak $\mathrm{C}-\mathrm{H} \cdots \mathrm{O}, \mathrm{H} \cdots \mathrm{H}$ and $\mathrm{H} \cdots \mathrm{C}$ interactions between DMF molecules, as shown by Hirshfeld surface analysis.

\section{Chemical context}

Extended hybrid organic-inorganic materials composed by transition metals and bridging carboxylates are interesting compounds that include the well-known metal-organic frameworks (MOFs), coordination polymers (CP) and coordination networks $(\mathrm{CN})$ (Batten et al., 2013). In the last decade, much of the research into this kind of compounds has focused in the design of materials looking for tunability of potential industrial applications such as lithium-ion batteries (Shin et al., 2015; Maiti et al., 2015; Tian et al., 2016), substitutes for dye-sensitized solar cells (DSSCs) (Zhang et al., 2018; Yan et al., 2018; Jeevadason et al., 2014), luminescent compounds (Kara et al., 2018; Igoa et al., 2019) and magnetic materials (Mesbah et al., 2014) among others. In the search for new extended hybrid materials based on $\mathrm{Ni}$ and terephthalate (terephthalate $=\mathrm{tp}=$ benzene-1,4-dicarboxylate), the title compound $\left[\mathrm{Ni}_{3}\left(\mathrm{C}_{8} \mathrm{H}_{4} \mathrm{O}_{4}\right)_{3}\left(\mathrm{C}_{3} \mathrm{H}_{7} \mathrm{NO}\right)_{4}\right]$ was synthesized by a solvothermal process in dimethylformamide (DMF) and is currently under study for application as an anode material in lithium-ion batteries. In order to perform an adequate structure-property correlation, the crystal structure of the compound was determined and supramolecular features of potential interest for understanding Li-ion intercalation and migration were analysed using the Hirshfeld surface (HS). 


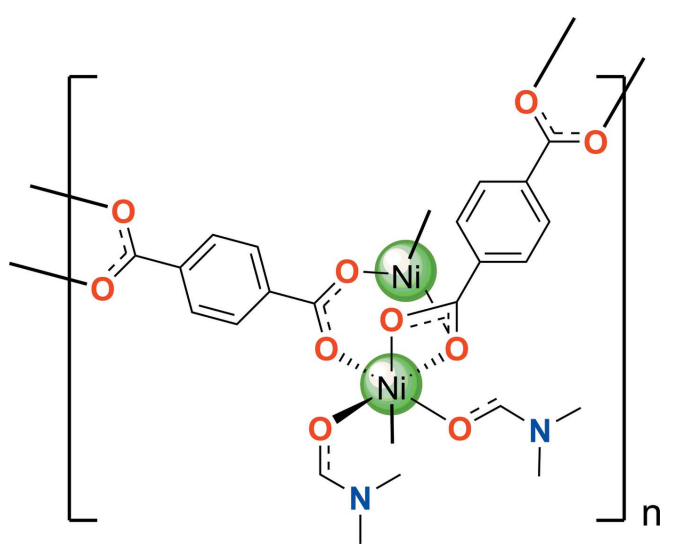

\section{Structural commentary}

The title compound is a two-dimensional coordination polymer formed by linear trinuclear centrosymmetric $\mathrm{Ni}_{3}(\mathrm{tp})_{3}(\mathrm{DMF})_{4}$ units connected through tp anions, which crystallizes in the monoclinic $P 2_{1} / c$ space group. Two distinct hexacoordinated $\mathrm{Ni}^{2+}$ cations (Ni1 in a special position with occupancy factor 0.5 ), two DMF ligands and two tp anions (anion $B$ in a special position with occupancy factor 0.5 ) exist in the asymmetric unit (Fig. 1). The central Ni atom, located on an inversion centre, displays an octahedral coordination to $\mathrm{O}$ atoms from three pairs of carboxylate units belonging to three symmetry-related tp anions with $\mathrm{Ni} 1-\mathrm{O}$ bond distances in the range 2.0205 (14)-2.0868 (14) $\AA$ and a maximum deviation of $4.85^{\circ}$ from the expected $\mathrm{O}-\mathrm{Ni1}-\mathrm{O}$ octahedral bond angles. The two terminal $\mathrm{Ni} 2$ cations also coordinate the carboxylate units of three symmetry-related tp units, one of them in bidentate mode, and two independent dimethylformamide ligands (one of them showing positional disorder) in a significantly distorted octahedron (Fig. 2). Ni2-O bond

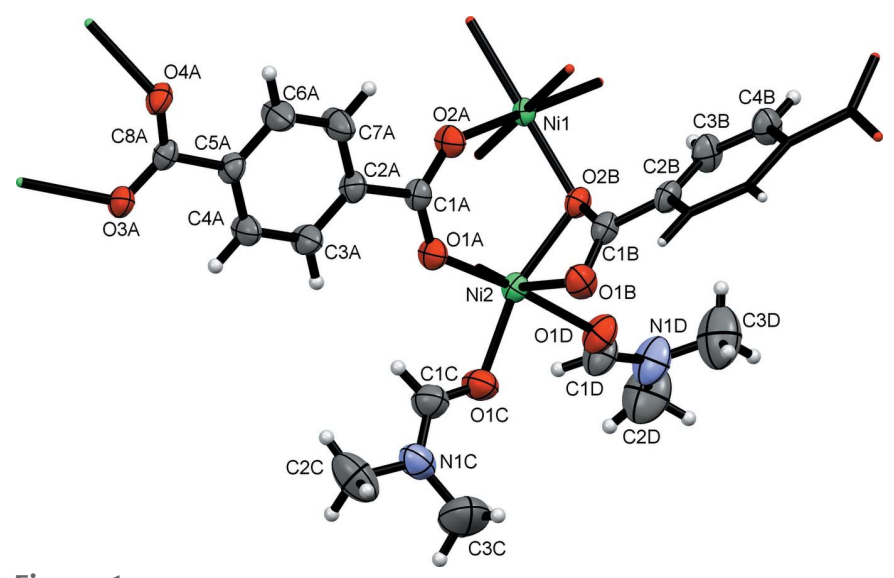

Figure 1

ORTEP model view of the asymmetric unit of $\left[\mathrm{Ni}_{3}\left(\mathrm{C}_{8} \mathrm{H}_{4} \mathrm{O}_{4}\right)_{3}\left(\mathrm{C}_{3} \mathrm{H}_{7} \mathrm{NO}\right)_{4}\right]$. Displacement ellipsoids are represented at the $50 \%$ probability level. Atoms completing the connectivity of those in the asymmetric unit and half symmetry-equivalent tp anion are shown as coloured spheres of arbitrary radii. Only one position of the disordered DMF ligand is shown for clarity.

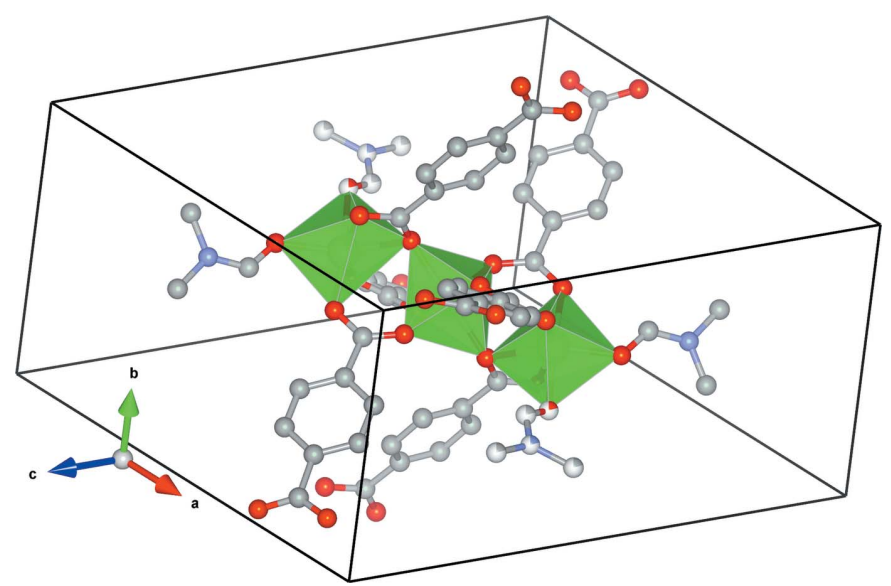

Figure 2

One trinuclear unit of $\left[\mathrm{Ni}_{3}\left(\mathrm{C}_{8} \mathrm{H}_{4} \mathrm{O}_{4}\right)_{3}\left(\mathrm{C}_{3} \mathrm{H}_{7} \mathrm{NO}\right)_{4}\right]$ highlighting the coordination polyhedra around each $\mathrm{Ni}$ atom and the coordination modes of the tp anions. Only one position of the disordered DMF ligand is shown and $\mathrm{H}$ atoms are omitted for clarity.

distances are in the range $2.0090(15)-2.0791$ (15) $\AA$ for terephthalate and 2.042 (12)-2.1853 (16) $\AA$ for DMF oxygen atoms respectively (including the lower occupancy disordered ligands). The $\mathrm{O} 1 B-\mathrm{Ni} 2-\mathrm{O} 2 B$ angle of $61.52(6)^{\circ}$ corresponding to a tridentate carboxylate, acting as bidentate towards $\mathrm{Ni} 2$, is very far away for the expected octahedral $90^{\circ}$ angle. However, the coordination is still octahedral since $\mathrm{O} 1 B$, $\mathrm{O} 2 B, \mathrm{O} 1 C$ and $\mathrm{O} 3 A$ form a clear equatorial plane with $\mathrm{Ni}$ deviating by just 0.1202 (7) $\AA$ from the plane and the rest of the equatorial bond angles $[\mathrm{O} 2 B-\mathrm{Ni} 2-\mathrm{O} 3 A=99.18(6)$, $\mathrm{O} 3 A-\mathrm{Ni} 2-\mathrm{O} 1 C=99.13(7)$ and $\mathrm{O} 1 C-\mathrm{Ni} 2-\mathrm{O} 1 B=$ $\left.98.91(7)^{\circ}\right]$ are increased by about $10^{\circ}$ to compensate for the very small angle from the bidentate ligand ( $\mathrm{O} 3 A$ is in position $\left.\frac{1}{2}+x, \frac{3}{2}-y, \frac{1}{2}+z\right)$. Additionally the two apical atoms $\mathrm{O} 1 A$ and $\mathrm{O} 1 D$ lie 2.026 (6) and 2.1269 (16) $\AA$, respectively, from the equatorial plane, forming an $\mathrm{O} 1 B D-\mathrm{Ni} 2-\mathrm{O} 1 A$ angle of $176.0(6)^{\circ}$. The carboxylate that is bidentate towards Ni2 is also monodentate towards Ni1, with the $\mathrm{O} 2 B$ atom being the link between corner-sharing Ni1 and Ni2 octahedra, which explains the longer $\mathrm{Ni}-\mathrm{O} 2 B$ bond distances of 2.0868 (14) and 2.0791 (15) $\AA$ to Ni1 and Ni2 respectively, compared with all other $\mathrm{Ni}-\mathrm{O}_{\mathrm{tp}}$ bond distances (see Fig. 2). The trinuclear octahedral arrangement with the three $\mathrm{Ni}$ atoms coordinated exclusively by $\mathrm{O}$ has only been observed in one 1,3benzenedicarboxylate catena-[bis $\left(\mu_{4^{-}}\right.$-isophthalato $) \operatorname{bis}\left(\mu_{3^{-}}\right.$ isophthalato)trinickel(II) bis(3-ethyl-1-methyl-1 $H$-imidazol3-ium)] (Chen et al., 2011) where the Ni cations are connected through the same number and coordination modes of carboxylate moieties. In that compound, however, two additional carboxylates complete the coordination spheres of the terminal Ni cations, instead of DMF molecules, giving a threedimensional connected network. Ni1 $\cdots \mathrm{Ni} 2$ distances of 3.4414 (4) $\AA$ are observed, also found in the 1,2-benzenedicarboxylate $\left(\mathrm{Ni}_{\text {central }} \cdots \mathrm{Ni}_{\text {terminal }}=3.442 \AA\right)$. This coordination mode is frequently found in other trinuclear transition metal carboxylates, with and without different ligands bonded to the terminal cations. 


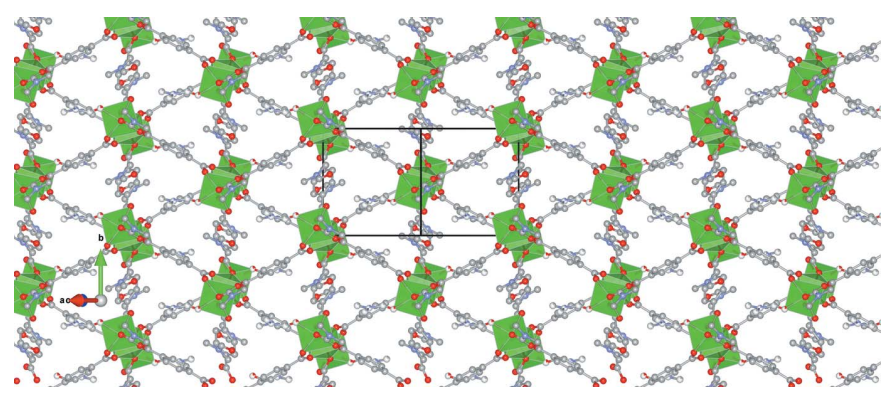

Figure 3

One plane of $\left[\mathrm{Ni}_{3}\left(\mathrm{C}_{8} \mathrm{H}_{4} \mathrm{O}_{4}\right)_{3}\left(\mathrm{C}_{3} \mathrm{H}_{7} \mathrm{NO}\right)_{4}\right]$ showing the hexagonal arrangement of equivalent units with slightly distorted distances. The tilt of the trinuclear octahedral units is also visible. $\mathrm{H}$ atoms and disordered positions of the DMF ligand have been omitted for clarity.

Each terephthalate ion links two nearby trinuclear units forming a slightly distorted two-dimensional hexagonal arrangement along the crystallographic (101) plane as shown in Fig. 3. Since the central $\mathrm{Ni}$ atom (Ni1) of the trinuclear arrangement is located at $(0,0,0)$ and equivalent $\left(\frac{1}{2}, \frac{1}{2}, \frac{1}{2}\right)$ coordinates, the hexagonal arrangement shows a $2+4$ distance pattern with two opposite nearby units at 9.6335 (11) $\AA$ (equal to the $b$-axis length) and four at 10.1407 (9) $\AA$ (equal to half of the short body diagonal of the unit cell) defining isosceles triangles with one small $\left[56.718(8)^{\circ}\right]$ and two larger $\left[\left(61.641(4)^{\circ}\right]\right.$ angles. The tp anions link nearby units in two different modes. The longest interunit distance corresponds to tp anions connecting the top or bottom parts of the unit, parallel to the plane (terephthalate anion $A$ ), while the shorter distance corresponds to a tp unit that is located over a centre of symmetry (anion $B$ ), which connects the top/bottom part of one unit to the bottom/top part of the next unit. This diagonal connection produces a tilt in the linear trinuclear units that are rotated by $11.82^{\circ}$ from the normal to the plane of the network, in a direction slightly away from the $b$ axis.

The ordered DMF molecules (labelled $C$ ) point outwards at both sides of the planes providing a polar surface that allows for the interaction of parallel planes of the coordination

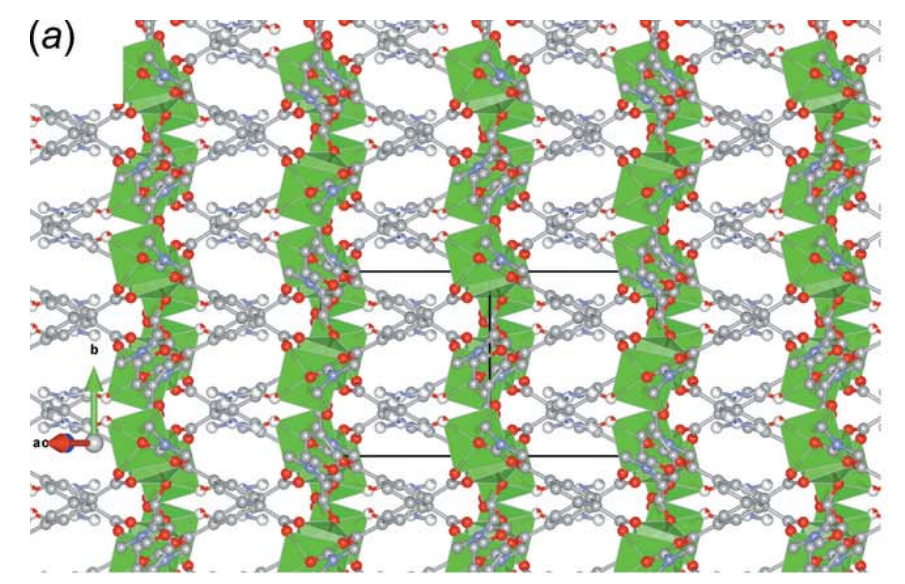

Table 1

Hydrogen-bond geometry $\left(\AA,^{\circ}\right)$.

\begin{tabular}{lllll}
\hline$D-\mathrm{H} \cdots A$ & $D-\mathrm{H}$ & $\mathrm{H} \cdots A$ & $D \cdots A$ & $D-\mathrm{H} \cdots A$ \\
\hline $\mathrm{C} 1 C-\mathrm{H} 1 C \cdots \mathrm{O} 1 A$ & 0.93 & 2.33 & $2.893(3)$ & 119 \\
$\mathrm{C} 2 C-\mathrm{H} 2 C B \cdots \mathrm{O} 1 B^{\mathrm{i}}$ & 0.96 & 2.56 & $3.367(5)$ & 143 \\
$\mathrm{C} 1 A D-\mathrm{H} 1 A D \cdots \mathrm{O} 2 B$ & 0.93 & 2.49 & $3.013(7)$ & 116 \\
$\mathrm{C} 2 A D-\mathrm{H} 2 D E \cdots \mathrm{O} 1 B^{\mathrm{ii}}$ & 0.96 & 2.39 & $3.089(8)$ & 130 \\
$\mathrm{C} 2 B D-\mathrm{H} 2 D G \cdots \mathrm{O} 1 B^{\mathrm{ii}}$ & 0.96 & 2.39 & $2.981(10)$ & 119 \\
\hline
\end{tabular}

Symmetry codes: (i) $-x+\frac{3}{2}, y+\frac{1}{2},-z+\frac{1}{2}$; (ii) $x+\frac{1}{2},-y+\frac{1}{2}, z+\frac{1}{2}$.

polymer. The disordered DMF ligands (labelled $D$ ) occupy part of the void space between consecutive planes (see Section 3 ) and were modelled over three different positions rotated by $180^{\circ}$ and displaced respectively, which strongly suggests that both static and dynamic disorder are present.

\section{Supramolecular features and Hirshfeld surface analysis}

Parallel planes do not stack in a typical hexagonal arrangement, where a layer projects over the voids of the poly[tris $\left(\mu_{4^{-}}\right.$ benzene-1,4-dicarboxylato)tetrakis(dimethylformamide)trinickel(II)], but in this case one layer projects over the center of the short inter-unit distance of the next layer, or is shifted by half of the $b$-axis length. This is again a consequence of the position of the Ni1 atoms at the corners and the centre of the unit cell, forming planes along (101). Fig. $4 a$ shows two parallel planes along the [101] direction (compare with Fig. 3) where it is shown that the projection of one plane falls away from the voids in the next one. Fig. $4 b$ shows the same two planes along the [010] direction where the relative position of the ordered DMF ligands in consecutive layers is shown.

In order to visualize the interplanar interactions, Hirshfeld surface (HS) analysis (Hirshfeld, 1977; Spackman \& Jayatilaka, 2009) was performed by using Crystal Explorer 17.5 (Turner et al., 2017). In the HS plotted over $d_{\text {norm }}$ (Fig. 5), the white surfaces indicate contacts with distances equal to the

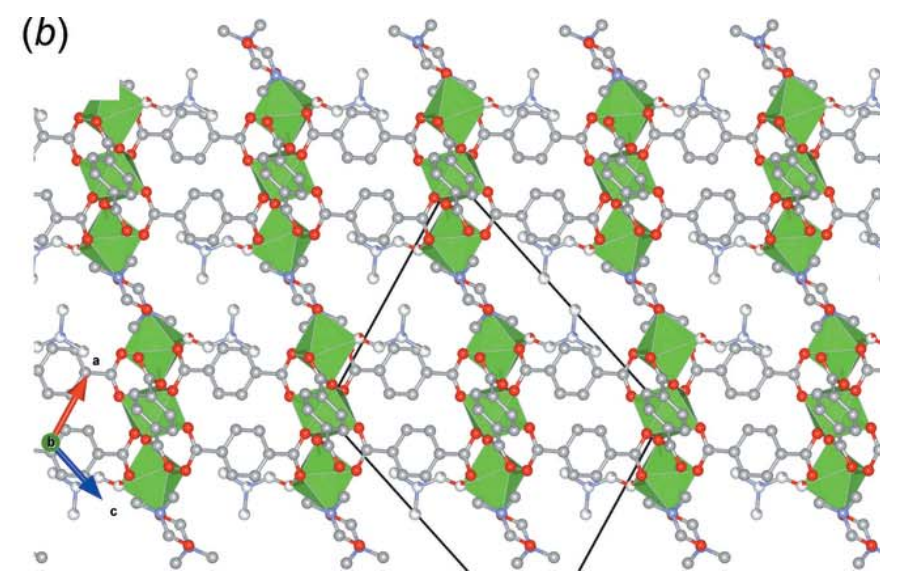

Figure 4

(a) View of two planes of the $\left[\mathrm{Ni}_{3}\left(\mathrm{C}_{8} \mathrm{H}_{4} \mathrm{O}_{4}\right)_{3}\left(\mathrm{C}_{3} \mathrm{H}_{7} \mathrm{NO}\right)_{4}\right]$ coordination polymer along [101] showing that the projection of one plane is shifted by $b / 2$ with respect to the next one and $(b)$ the same two planes projected along [010] showing the relative position of ordered and disordered DMF molecules with respect to the planes. 
sum of van der Waals radii, and the red and blue colours indicate distances shorter (in close contact) or longer (distant contact) than the van der Waals radii, respectively (Venkatesan et al., 2016). Since bonds from Ni1 to $\mathrm{O}$ atoms and from $\mathrm{C} 2 \mathrm{~B}$ and $\mathrm{C} 4 \mathrm{~B}$ to $\mathrm{C}$ atoms are not included in the asymmetric unit, bright-red spots appear over them. The following stronger short contacts shown as light-red spots correspond to weak $\mathrm{C}-\mathrm{H} \cdots \mathrm{O}$ hydrogen bonds shown in Table 1 . It is interesting to note that the ordered DMF- $C$ molecule shows one intramolecular $\mathrm{C} 1 C-\mathrm{H} 1 C \cdots \mathrm{O} 1 A$ and one interplanar $\mathrm{C} 2 C-\mathrm{H} 2 \mathrm{C} B \cdots \mathrm{O} 1 B^{\mathrm{i}}$ hydrogen bond [symmetry code: (i) $\left.-x+\frac{3}{2}, y+\frac{1}{2},-z+\frac{1}{2}\right]$. The former limits the rotation of the DMF group and the latter the orientation. This fixes the DMF- $C$ molecules and provides the main interaction between parallel network planes. The DMF- $D$ molecule, disordered over three positions, participates in no hydrogen bonds to the aldehyde carbon $(\mathrm{C} 1 D, \mathrm{C} 1 A D$ or $\mathrm{C} 1 B D)$ but only to methyl $\mathrm{H}$ atoms, giving the molecule rotational freedom. Additionally, the DMF molecule is smaller than the void in which it sits, allowing for additional positional freedom. Removing DMF-C and DMF- $D$ from the structural model, allowed the volume these molecules occupy in the crystal structure to be calculated. The void-calculation routine in PLATON (Spek, 2009) was used, with a probe radius of $1.2 \AA$ (enough to place small monoatomic ions). Voids arising from removing DMF- $C$ and DMF- $D$ are 110.18 and $167.93 \AA^{3}$ per molecule, respectively (two of the voids are connected around $\frac{1}{4},-0.07, \frac{3}{4}$ and $\frac{3}{4},-0.02, \frac{1}{4}$ for DMF- $C$ and $1 / 2,0.003,0$ and $0,0.496,1 / 2$ for DMF$D$ ), again showing that the DMF- $D$ molecule is located over a much larger void than its own size, justifying the observation of positional disorder. Moreover, performing the same void calculation procedure using each of the DMF- $D$ positions individually (as is the real case for each appearance of the molecule in the crystal), it is observed that the highest occupied position of DMF- $D$ leaves only $21.75 \AA^{3}$ free volume per molecule (in two smaller $10.88 \AA^{3}$ voids) while DMF- $A D$ and DMF- $B D$ leave larger 53.1 and $37.7 \AA^{3}$ voids, respectively. Besides the described hydrogen-bond interactions, contacts between $\mathrm{H}$ atoms from both DMF molecules and neigh-

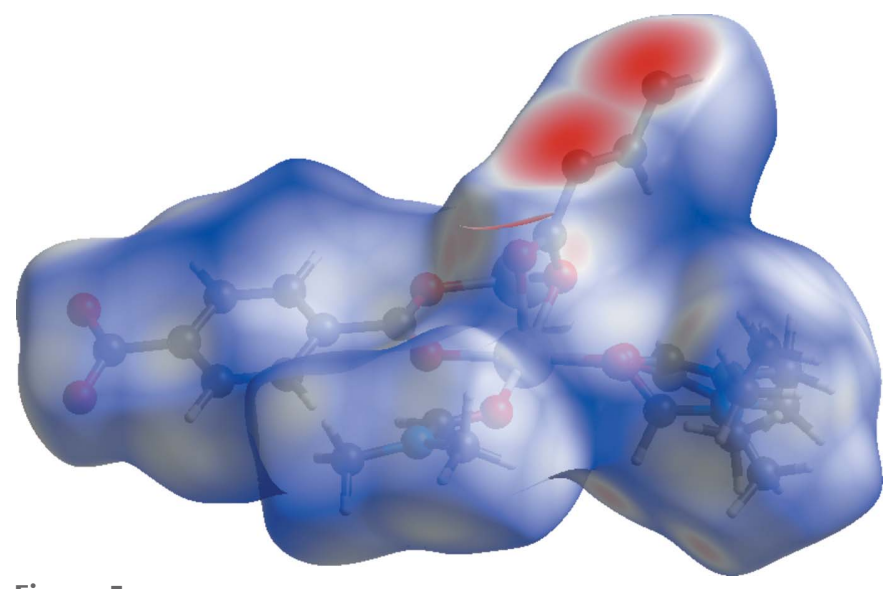

Figure 5

View of the three-dimensional Hirshfeld surface of the title complex plotted over $d_{\text {norm }}$ in the range -0.7548 to 1.5398 a.u.
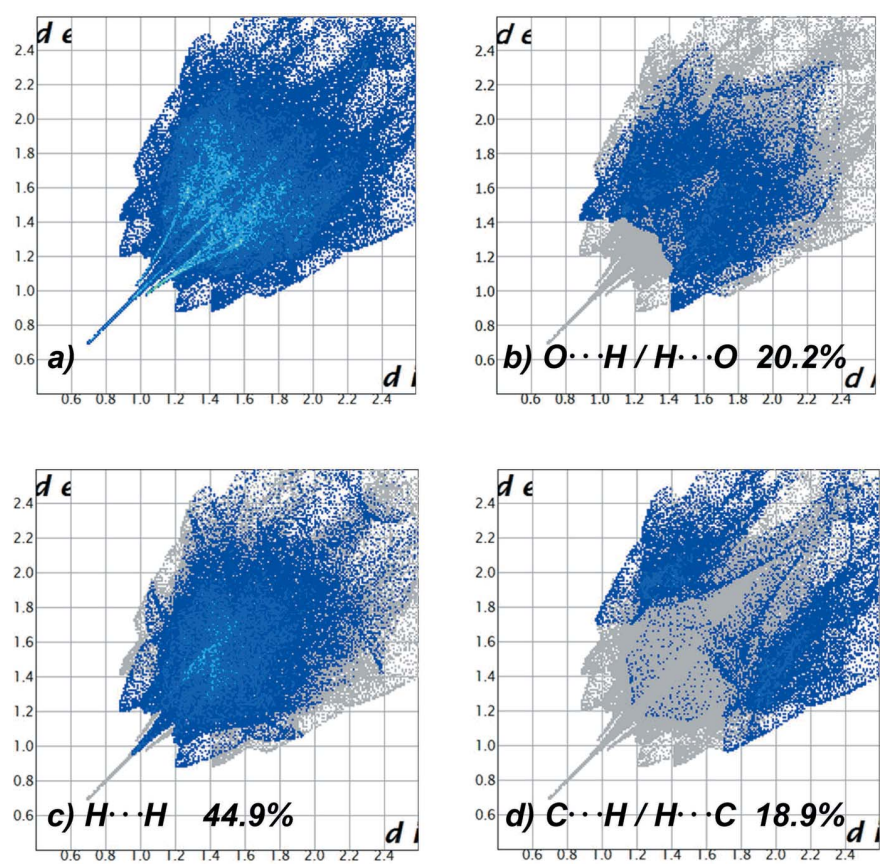

Figure 6

Two-dimensional fingerprint plot of the total (a) (top left) and specific $(b)$ $\mathrm{O} \cdots \mathrm{H} / \mathrm{H} \cdots \mathrm{O}$ (top right), (c) $\mathrm{H} \cdots \mathrm{H}$, (bottom left) and $(d) \mathrm{C} \cdots \mathrm{H} / \mathrm{H} \cdots \mathrm{C}$ (bottom right) interactions in $\left[\mathrm{Ni}_{3}\left(\mathrm{C}_{8} \mathrm{H}_{4} \mathrm{O}_{4}\right)_{3}\left(\mathrm{C}_{3} \mathrm{H}_{7} \mathrm{NO}\right)_{4}\right]$. Note that $\mathrm{H} \cdots \mathrm{H}$ interactions coming from the methyl residues of DMF ligands are dominant and define the most relevant interactions among consecutive layers of the compound.

bouring $\mathrm{H}, \mathrm{O}$ and $\mathrm{C}$ atoms from surrounding DMF and tp anions dominate the interactions in the crystal structure, as depicted in Fig. 6, where the two-dimensional fingerprint plots (McKinnon et al., 2007) are shown. $\mathrm{H} \cdots \mathrm{H}$ interactions from the DMF ligands are the most relevant, covering $45 \%$ of the Hirshfeld surface The presence of voids and a significant number of weak interlayer interactions may well explain the possibility of using this material for Li-ion batteries, as will be discussed elsewhere.

\section{Database survey}

The May 2019 update of the CSD (Groom et al., 2016) contains six coordination networks comprising $\mathrm{Ni}$ and a terephthalate anion as the sole linker; however, none of them contains only $\mathrm{O}$ in the coordination sphere. Additionally, there are eight trinuclear linear $\mathrm{Ni}$ compounds formed by carboxylates and other oxygenated ligands, none of them coordination networks except for DAFHID (Chen et al., 2011), which is discussed above.

\section{Synthesis and crystallization}

The compound was synthesized by solvothermal method via reaction between $\mathrm{NiCl}_{2} \cdot 6 \mathrm{H}_{2} \mathrm{O}(0.6143 \mathrm{~g}, 2.58 \mathrm{mmol})$, terephthalic acid $(0.8587 \mathrm{~g}, 5.20 \mathrm{mmol})$ and $N, N$-dimethylformamide 
$(\mathrm{DMF})(50 \mathrm{ml})$ as a solvent; the reactants were dissolved in DMF and transferred to a steel autoclave at $423 \mathrm{~K}$ for $24 \mathrm{~h}$.

The green crystals were collected by filtration, washed several times with DMF and dried at $373 \mathrm{~K}$ (yield $0.7 \mathrm{mg}$, $70 \%)$. Elemental Analysis for $\mathrm{Ni}_{3}\left(\mathrm{C}_{8} \mathrm{H}_{4} \mathrm{O}_{4}\right)_{3}\left(\mathrm{C}_{3} \mathrm{H}_{7} \mathrm{NO}\right)_{4}\left(M_{\mathrm{r}}=\right.$ 960.81). Calculated (\%): C 45.00, H 4.20, N 5.83, Ni 18.33 . Found: C44.95, H 4.21, N 5.85, Ni 18.22.

\section{Refinement}

Crystal data, data collection and structure refinement details are summarized in Table 2. All hydrogen atoms were placed at geometrically suitable positions and refined riding with $U_{\text {iso }}(\mathrm{H})=1.2$ or 1.5 times the $U_{\text {eq }}$ of the parent C atom. There are two sites occupied with $N, N$-dimethylformamide (DMF) molecules; one of them showing disorder that was modelled in three different positions with relative occupancies of 0.502 , 0.286 and 0.212 . This causes $\mathrm{C}$ atoms from the DMF methyl groups to have very large thermal displacement parameters that required the use of similarity restraints to converge to reasonable values.

\section{Acknowledgements}

This work is part of the PhD thesis of CAC (University of KTH) on Hybrid Materials for lithium batteries. The authors acknowledge the organizers of the 1st LACA School on Small Molecule Crystallography (Latin American Crystallographic Association) in Montevideo, Uruguay, for providing access to the single-crystal and powder X-ray diffractometers used to determine this structure.

\section{Funding information}

Funding for this research was provided by: ASDI (Swedish International Development Agency); KHT (Royal Institute of Technology) University, Department of Applied Electrochemistry; UMSA (Universidad Mayor de San Andrés), Departament of Inorganic Chemistry and Materials Science/ Advanced Materials, IIQ Chemical Research Institute; PEDECIBA Química (Uruguay) (grant to L. Suescun).

\section{References}

Batten, S. R., Champness, N. R., Chen, X.-M., Garcia-Martinez, J., Kitagawa, S., Öhrström, L., O'Keeffe, M., Paik Suh, M. \& Reedijk, J. (2013). Pure Appl. Chem. 85, 1715-1724.

Bruker (2014). APEX2 and SAINT. Bruker AXS Inc., Madison, Wisconsin, USA.

Chen, W.-X., Zhuang, G.-L., Zhao, H.-X., Long, L.-S., Huang, R.-B. \& Zheng, L.-S. (2011). Dalton Trans. 40, 10237-10241.

Groom, C. R., Bruno, I. J., Lightfoot, M. P. \& Ward, S. C. (2016). Acta Cryst. B72, 171-179.

Hirshfeld, H. L. (1977). Theor. Chim. Acta, 44, 129-138.

Igoa, F., Peinado, G., Suescun, L., Kremer, C. \& Torres, J. (2019). J. Solid State Chem. In the Press.

Jeevadason, W. A., Murugavel, K. \& Neelakantan, M. A. (2014). Renew. Sustain. Energy Rev. 36, 220-227.

Kara, D. A., Donmez, A., Kara, H. \& Coban, M. B. (2018). Acta Cryst. C74, 901-906.
Table 2

Experimental details.

\begin{tabular}{ll}
\hline Crystal data & \\
Chemical formula & {$\left[\mathrm{Ni}_{3}\left(\mathrm{C}_{8} \mathrm{H}_{4} \mathrm{O}_{4}\right)_{3}\left(\mathrm{C}_{3} \mathrm{H}_{7} \mathrm{NO}\right)_{4}\right]$} \\
$M_{\mathrm{r}}$ & 960.85 \\
Crystal system, space group & Monoclinic, $P 2_{1} / n$ \\
Temperature (K) & 298 \\
$a, b, c(\AA)$ & $14.0309(16), 9.6335(11)$, \\
& $16.5804(19)$ \\
$\beta\left({ }^{\circ}\right)$ & $109.230(5)$ \\
$V\left(\AA^{3}\right)$ & $2116.1(4)$ \\
$Z$ & 2 \\
Radiation type & $\mathrm{Cu} \mathrm{K \alpha}$ \\
$\mu\left(\mathrm{mm}^{-1}\right)$ & 2.18 \\
Crystal size $(\mathrm{mm})$ & $0.18 \times 0.14 \times 0.08$
\end{tabular}

Data collection

Diffractometer

Absorption correction

$T_{\min }, T_{\max }$

No. of measured, independent and observed $[I>2 \sigma(I)]$ reflections

$R_{\text {int }}$

$(\sin \theta / \lambda)_{\max }\left(\AA^{-1}\right)$

Bruker D8 Venture

Multi-scan ( $S A D A B S$; Krause $e t$ al., 2015)

$0.657,0.754$

21976, 4170, 3539

0.040

0.619

Refinement

$R\left[F^{2}>2 \sigma\left(F^{2}\right)\right], w R\left(F^{2}\right), S$

No. of reflections

No. of parameters

No. of restraints

$\mathrm{H}$-atom treatment

$\Delta \rho_{\max }, \Delta \rho_{\min }\left(\mathrm{e} \AA^{-3}\right)$

$0.033,0.092,1.04$

4170

339

353

$\mathrm{H}$-atom parameters constrained $0.28,-0.52$

Computer programs: APEX2 and SAINT (Bruker, 2014), SHELXT (Sheldrick, 2015a), SHELXL2018/3 (Sheldrick, 2015b), Mercury (Macrae et al., 2008), VESTA (Momma \& Izumi, 2011) and publCIF (Westrip, 2010).

Krause, L., Herbst-Irmer, R., Sheldrick, G. M. \& Stalke, D. (2015). J. Appl. Cryst. 48, 3-10.

Macrae, C. F., Bruno, I. J., Chisholm, J. A., Edgington, P. R., McCabe, P., Pidcock, E., Rodriguez-Monge, L., Taylor, R., van de Streek, J. \& Wood, P. A. (2008). J. Appl. Cryst. 41, 466-470.

Maiti, S., Pramanik, A., Manju, U. \& Mahanty, S. (2015). Appl. Mater. Interfaces, 7, 16357-16363.

McKinnon, J. J., Jayatilaka, D. \& Spackman, M. A. (2007). Chem. Commun. 3814-3816.

Mesbah, A., Rabu, P., Sibille, R., Lebègue, S., Mazet, T., Malaman, B. \& François, M. (2014). Inorg. Chem. 53, 872-881.

Momma, K. \& Izumi, F. (2011). J. Appl. Cryst. 44, 1272-1276.

Shin, J., Kim, M., Cirera, J., Chen, S., Halder, G. J., Yersak, T. A., Paesani, F., Cohen, S. F. \& Meng, J. S. (2015). J. Mater. Chem. A, 7, 4259-4290.

Sheldrick, G. M. (2015). Acta Cryst. A71, 3-8.

Sheldrick, G. M. (2015). Acta Cryst. C71, 3-8.

Spackman, M. A. \& Jayatilaka, D. (2009). CrystEngComm, 11, 1932.

Spek, A. L. (2009). Acta Cryst. D65, 148-155.

Tian, B., Ning, G.-H., Gao, Q., Tan, L.-M., Tang, W., Chen, Z., Su, C. \& Loh, K. P. (2016). Appl. Mater. Interfaces, 8, 31067-31075.

Turner, M. J., McKinnon, J. J., Wolff, S. K., Grimwood, D. J., Spackman, P. R., Jayatilaka, D. \& Spackman, M. A. (2017). Crystal Explorer 17.5. The University of Western Australia.

Venkatesan, P., Thamotharan, S., Ilangovan, A., Liang, H. \& Sundius, T. (2016). Spectrochim. Acta Part A, 153, 625-636.

Westrip, S. P. (2010). J. Appl. Cryst. 43, 920-925.

Yan, L., Li, R., Shen, W. \& Qi, Z. (2018). J. Lumin. 194, 151-155.

Zhang, J., Xu, L. \& Wong, W. Y. (2018). Coord. Chem. Rev. 355, 180198. 


\section{supporting information}

Acta Cryst. (2019). E75, 1839-1843 [https://doi.org/10.1107/S2056989019014658]

Crystal structure and Hirshfeld surface analysis of poly[tris $\left(\mu_{4}\right.$-benzene-1,4-dicarboxylato)tetrakis(dimethylformamide)trinickel(II)]: a two-dimensional coordination network

Cesario Ajpi, Leopoldo Suescun, Naviana Leiva, Anders Lundblad, Göran Lindbergh and Saul

\section{Cabrera}

Computing details

Data collection: APEX2 (Bruker, 2014); cell refinement: SAINT (Bruker, 2014); data reduction: SAINT (Bruker, 2014); program(s) used to solve structure: SHELXT (Sheldrick, 2015a); program(s) used to refine structure: SHELXL2018/3 (Sheldrick, 2015b); molecular graphics: Mercury (Macrae et al., 2008) and VESTA (Momma \& Izumi, 2011); software used to prepare material for publication: publCIF (Westrip, 2010).

Poly[tris( $\mu_{4}$-benzene-1,4-dicarboxylato)tetrakis(dimethylformamide)trinickel(II)]

Crystal data

$\left[\mathrm{Ni}_{3}\left(\mathrm{C}_{8} \mathrm{H}_{4} \mathrm{O}_{4}\right)_{3}\left(\mathrm{C}_{3} \mathrm{H}_{7} \mathrm{NO}\right)_{4}\right]$

$F(000)=992$

$M_{r}=960.85$

Monoclinic, $P 2_{1} / n$

$a=14.0309(16) \AA$

$b=9.6335(11) \AA$

$c=16.5804$ (19) $\AA$

$\beta=109.230(5)^{\circ}$

$V=2116.1(4) \AA^{3}$

$D_{\mathrm{x}}=1.508 \mathrm{Mg} \mathrm{m}^{-3}$

$\mathrm{Cu} K \alpha$ radiation, $\lambda=1.54178 \AA$

$Z=2$

Cell parameters from 9446 reflections

$\theta=3.6-72.4^{\circ}$

$\mu=2.18 \mathrm{~mm}^{-1}$

$T=298 \mathrm{~K}$

Plate, green

$0.18 \times 0.14 \times 0.08 \mathrm{~mm}$

Data collection

Bruker D8 Venture

21976 measured reflections diffractometer

Radiation source: Incoatec microsource

Detector resolution: 10.25 pixels $\mathrm{mm}^{-1}$

$/ \mathrm{j}$ and /w scans

Absorption correction: multi-scan

(SADABS; Krause, et al., 2015)

$T_{\text {min }}=0.657, T_{\max }=0.754$

4170 independent reflections

3539 reflections with $I>2 \sigma(I)$

$R_{\text {int }}=0.040$

$\theta_{\text {max }}=72.5^{\circ}, \theta_{\min }=3.6^{\circ}$

$h=-16 \rightarrow 17$

$k=-11 \rightarrow 9$

$l=-20 \rightarrow 20$

Refinement

Refinement on $F^{2}$

Least-squares matrix: full

$R\left[F^{2}>2 \sigma\left(F^{2}\right)\right]=0.033$

$w R\left(F^{2}\right)=0.092$

$S=1.04$

4170 reflections

\section{9 parameters}

353 restraints

Hydrogen site location: inferred from neighbouring sites

$\mathrm{H}$-atom parameters constrained 
$w=1 /\left[\sigma^{2}\left(F_{\mathrm{o}}^{2}\right)+(0.0499 P)^{2}+0.8233 P\right]$

where $P=\left(F_{\mathrm{o}}^{2}+2 F_{\mathrm{c}}^{2}\right) / 3$

$(\Delta / \sigma)_{\max }=0.001$

Special details

Geometry. All esds (except the esd in the dihedral angle between two 1.s. planes) are estimated using the full covariance matrix. The cell esds are taken into account individually in the estimation of esds in distances, angles and torsion angles; correlations between esds in cell parameters are only used when they are defined by crystal symmetry. An approximate (isotropic) treatment of cell esds is used for estimating esds involving 1.s. planes.

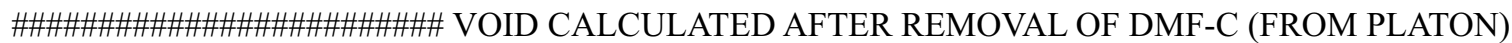
Search for and

Analysis of Solvent Accessible Voids in the Structure - Grid = 0.20 Ang., Probe Radius = 1.20 Ang., NStep = 6

:: Total Potential Solvent Area Vol 440.3 Ang**3 per Unit Cell Vol 2116.1 Ang**3 [20.8\%]

Area \#GridPoint VolPerc. Vol(A**3) X(av) Y(av) Z(av) Eigenvector(frac) Sig(Ang) 130203 [ 5658] 10220 [ 41.2] 0.250-0.068 0.750 1 0.125 1.000-0.2313.0020.2450.677

$1.0001 .8531 .000-0.3100 .1871 .20230205$ [5658] 10220 [ 41.2] 0.750-0.018 0.250 $10.1581 .0000 .0252 .912-0.042$ $0.0681 .0002 .0831 .000-0.2970 .3121 .17$

x y z Shortest Contacts within 4.5 Ang. (Excl. H)

0.250-0.068 0.750 C2D 2.97; O1A 3.18; Ni2 3.31; O1B 3.66; O3A 3.72; C3BD 3.99; C2BD 4.06; C3A 4.29; O1AD 4.30; 2 0.750-0.018 0.250 Ni2 3.06; O1A 3.19; C2D 3.23; O3A 3.86; O1AD 3.89; O1D 4.02; O1BD 4.07; O1B 4.07; C2BD 4.31 ; \#\#\#\#\#\#\#\#\#\#\#\#\#\#\#\#\#\#\#\#\#\# VOID CALCULATED AFTER REMOVAL OF DMF-D (FROM PLATON) Search for and Analysis of Solvent Accessible Voids in the Structure - Grid =0.20 Ang., Probe Radius = 1.20 Ang., NStep = 6

:: Total Potential Solvent Area Vol 671.7 Ang**3 per Unit Cell Vol 2116.1 Ang**3 [31.7\%]

Area \#GridPoint VolPerc. Vol(A**3) X(av) Y(av) Z(av) Eigenvector(frac) Sig(Ang) 146072 [ 11117] $16336[81.0] 0.5000 .0030 .0001-0.3931 .0000 .4913 .5620 .059-1.000$

0.6031 .5931 .0000 .4230 .4581 .49246084 [11117] $16336[81.0] 1.0000 .4960 .50010 .3931 .000-0.4903 .5620 .037$ $1.0000 .5891 .5931 .000-0.4010 .4711 .49$

x y z Shortest Contacts within 4.5 Ang. (Excl. H)

0.003 0.000 O3A 3.66; C4A 3.72; O1C 3.86; Ni2 4.37; C3A 4.46; 2 1.000 0.496 0.500 O3A 3.66; C4A 3.72; O1C 3.86; $\mathrm{Ni} 2$ 4.37; C3A 4.46;

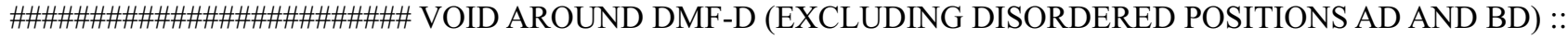
Total Potential Solvent Area Vol 43.5 Ang**3 per Unit Cell Vol 2116.1 Ang**3 [ 2.1\%] Area \#GridPoint VolPerc. Vol(A**3) X(av) Y(av) Z(av) Eigenvector(frac) Sig(Ang) 11492 [5] 1 11[0.0] 0.368 0.018 0.206 1 -0.079-0.096 1.000 0.6320.359 1.000 0.1500.62 3 -1.000 0.684-0.306 0.592 1492[5] 1 11[0.0] 0.632-0.018 0.794 1 -0.082-0.079 1.000 0.63 20.3581 .0000 .1440 .623 -1.000 0.681-0.3120.593 1492[5] 1 11] 0.0 [ $0.8680 .4820 .7061-0.0620 .0341 .0000 .632-0.3811 .000-0.1320 .623$ $-1.000-0.722-0.3100 .5941491[5] 111[0.0] 0.1320 .5180 .2941-0.038-0.0481 .0000 .632-0.3941 .000-0.1030 .623$ 
$-1.000-0.744-0.3150 .59$

x y z Shortest Contacts within 4.5 Ang. (Excl. H)

10.368

0.018 0.206 N1D 2.91; C5A 3.04; C4A 3.14; C3D 3.23; C7A 3.24; C2D 3.25; C1D 3.30; C6A 3.31; C4B 3.33; 2

0.632-0.018 0.794 N1D 2.91; C5A 3.04; C4A 3.15; C3D 3.23; C7A 3.24; C2D 3.24; C1D 3.30; C6A 3.31; C4B 3.33; 3

0.868 0.482 0.706 N1D 2.91; C5A 3.04; C4A 3.15; C3D 3.23; C7A 3.23; C2D 3.25; C1D 3.30; C6A 3.31; C4B 3.33;

40.1320 .518

0.294 N1D 2.91; C5A 3.04; C4A 3.14; C3D 3.23; C7A 3.24; C2D 3.25; C1D 3.30; C6A 3.31; C4B 3.33;

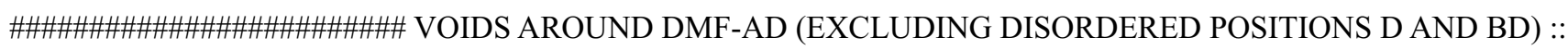

Total Potential Solvent Area Vol 106.2 Ang**3 per Unit Cell Vol 2116.1 Ang**3 [ 5.0\%]

Area \#GridPoint VolPerc. Vol(A**3) X(av) Y(av) Z(av) Eigenvector(frac) Sig(Ang)

- 1 5282[133] 2 39[1.0] $0.5000 .0001 .00010 .349-0.2551 .0001 .5521 .000-0.0190 .030$

0.8130 .0431 .0000 .0970 .682998 [ 1 [ 0 7 [ 0.0 [ $0.7500 .0420 .57111 .000-0.023-0.1640 .5520 .580-0.0511 .0000 .543$ $-0.020-1.000-0.0170 .5235291[133] 239[1.0] 1.0000 .5000 .50010 .3490 .2551 .0001 .5521 .0000 .0070 .0310 .813$ $-0.0371 .000-0.0970 .684998[1] 07$ [ 0.0 [ $0.2500 .4580 .07111 .000-0.017-0.1700 .5520 .587-0.0461 .0000 .543$ $-0.017-1.000-0.0160 .525998[1] 07$ [ 0.0$] 0.7500 .5420 .92911 .000-0.032-0.1570 .5520 .571-0.1191 .0000 .543$ $-0.038-1.000-0.0420 .526998[1] 07[0.0] 0.2500 .9580 .42911 .000-0.024-0.1690 .5520 .587-0.0021 .0000 .543$ $-0.010-1.0000 .0010 .52$

x y z Shortest Contacts within 4.5 Ang. (Excl. H) 10.500

0.000 1.000 C3AD 3.20; O1AD 3.26; N1AD 3.29; C1AD 3.37; O3A 3.66; C4A 3.74; O1C 3.87; C2AD 4.26; Ni2 4.37; 2 0.750 0.042 0.571 C3B 2.91; C1AD 2.92; C6A 2.98; C2B 3.02; C2C 3.02; C5A 3.03; N1AD 3.08; C7A 3.13; C4A 3.21; 3 1.000 0.500 0.500 C3AD 3.20; O1AD 3.26; N1AD 3.29; C1AD 3.37; O3A 3.66; C4A 3.74; O1C 3.87; C2AD 4.26; Ni2 4.37; 40.250 0.458 0.071 C3B 2.91; C1AD 2.91; C6A 2.98; C2B 3.02; C2C 3.02; C5A 3.03; N1AD 3.08; C7A 3.13; C4A 3.21; 50.750 0.542 0.929 C3B 2.91; C1AD 2.92; C6A 2.98; C2B 3.02; C2C 3.02; C5A 3.03; N1AD 3.08; C7A 3.13; C4A 3.21; 60.2500 .958 0.429 C3B 2.91; C1AD 2.91; C6A 2.98; C2B 3.02; C2C 3.02; C5A 3.03; N1AD 3.07; C7A 3.13; C4A 3.21;

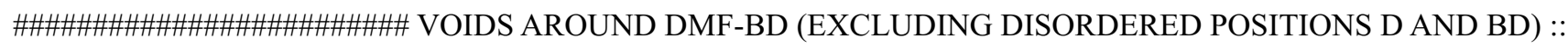
Total Potential Solvent Area Vol 75.4 Ang**3 per Unit Cell Vol 2116.1 Ang**3 [ 3.6\%]

Area \#GridPoint VolPerc. Vol(A**3) X(av) Y(av) Z(av) Eigenvector(frac) Sig(Ang) $12589[15] 119[0.1] 0.7660 .0880 .6181-0.1450 .9731 .0001 .0521 .0000 .2240 .2970 .66$ $3-0.1101 .000-0.3560 .6022584[15] 119[0.1] 0.2660 .4120 .1181-0.139-0.9651 .0001 .0521 .000-0.2420 .2890 .663$ $0.1231 .0000 .3580 .5932588[15] 119[0.1] 0.7340 .5880 .8821-0.143-0.9731 .0001 .0521 .000-0.2300 .2940 .663$ $0.1141 .0000 .3580 .6042585[15] 119[0.1] 0.2340 .9120 .3821-0.1470 .9671 .0001 .0521 .0000 .2070 .3040 .663$ $-0.0971 .000-0.3490 .60$

x y z Shortest Contacts within 4.5 Ang. (Excl. H) 10.766

0.088 0.618 C7A 2.93; C1BD 2.93; C3B 2.97; C2A 3.09; C6A 3.13; N1BD 3.18; O1BD 3.21; C2B 3.38; C3A 3.40; 2 0.266 0.412 0.118 C1BD 2.93; C7A 2.93; C3B 2.97; C2A 3.09; C6A 3.13; N1BD 3.17; O1BD 3.21; C2B 3.38; C3A 3.41; 30.7340 .588 0.882 C7A 2.93; C1BD 2.93; C3B 2.97; C2A 3.09; C6A 3.13; N1BD 3.17; O1BD 3.21; C2B 3.38; C3A 3.40; 40.2340 .9120 .382 C1BD 2.93; C7A 2.93; C3B 2.97; C2A 3.09; C6A 3.13; N1BD 3.17; O1BD 3.21; C2B 3.39; 
C3A 3.41;

Fractional atomic coordinates and isotropic or equivalent isotropic displacement parameters $\left(\AA^{2}\right)$

\begin{tabular}{|c|c|c|c|c|c|}
\hline & $x$ & $y$ & $z$ & $U_{\text {iso }} * / U_{\text {eq }}$ & Occ. $(<1)$ \\
\hline Ni1 & 0.500000 & 0.500000 & 0.500000 & $0.02455(12)$ & \\
\hline $\mathrm{Ni} 2$ & $0.67419(2)$ & $0.42716(4)$ & 0.40049 (2) & $0.03009(11)$ & \\
\hline O1A & $0.56051(11)$ & $0.52816(17)$ & $0.31290(9)$ & $0.0386(3)$ & \\
\hline $\mathrm{C} 1 \mathrm{~A}$ & $0.47483(15)$ & $0.5613(2)$ & $0.31483(12)$ & $0.0318(4)$ & \\
\hline $\mathrm{O} 2 \mathrm{~A}$ & $0.44086(11)$ & $0.54372(18)$ & $0.37410(9)$ & $0.0406(4)$ & \\
\hline $\mathrm{C} 2 \mathrm{~A}$ & $0.40430(15)$ & $0.6317(2)$ & $0.23606(13)$ & $0.0350(4)$ & \\
\hline $\mathrm{O} 3 \mathrm{~A}$ & $0.23759(11)$ & $0.90569(16)$ & $-0.03005(9)$ & 0.0380 & \\
\hline $\mathrm{C} 3 \mathrm{~A}$ & $0.43627(17)$ & $0.6769(3)$ & $0.17032(14)$ & $0.0453(6)$ & \\
\hline $\mathrm{H} 3 \mathrm{~A}$ & 0.502355 & 0.659985 & 0.172565 & $0.054 *$ & \\
\hline $\mathrm{O} 4 \mathrm{~A}$ & $0.11253(10)$ & $0.85882(16)$ & $0.02142(10)$ & 0.0384 & \\
\hline $\mathrm{C} 3 \mathrm{~B}$ & 0.54232 (19) & $0.1105(2)$ & $0.55325(15)$ & $0.0435(5)$ & \\
\hline H3B & 0.570430 & 0.184531 & 0.589193 & $0.052 *$ & \\
\hline C5A & $0.27238(15)$ & $0.7701(2)$ & 0.09543 (13) & $0.0340(4)$ & \\
\hline C6A & $0.23995(18)$ & 0.7236 & $0.16061(16)$ & $0.0550(7)$ & \\
\hline H6A & 0.173356 & 0.738276 & 0.157606 & $0.066^{*}$ & \\
\hline C7A & $0.30498(18)$ & $0.6555(3)$ & $0.23020(16)$ & $0.0568(7)$ & \\
\hline H7A & 0.281846 & 0.625248 & 0.273702 & $0.068 *$ & \\
\hline C8A & $0.20106(15)$ & $0.8513(2)$ & $0.02281(12)$ & $0.0315(4)$ & \\
\hline $\mathrm{C} 4 \mathrm{~A}$ & $0.37147(17)$ & $0.7469(3)$ & $0.10111(14)$ & $0.0445(6)$ & \\
\hline $\mathrm{H} 4 \mathrm{~A}$ & 0.394827 & 0.778659 & 0.058044 & $0.053 *$ & \\
\hline O1B & $0.59907(13)$ & $0.22615(17)$ & $0.36814(10)$ & $0.0447(4)$ & \\
\hline C1B & $0.57649(15)$ & $0.2365(2)$ & $0.43443(14)$ & $0.0349(4)$ & \\
\hline $\mathrm{O} 2 \mathrm{~B}$ & $0.59547(10)$ & $0.35001(14)$ & $0.47735(9)$ & 0.0323 & \\
\hline $\mathrm{C} 2 \mathrm{~B}$ & $0.53512(16)$ & $0.1155(2)$ & $0.46820(15)$ & $0.0364(5)$ & \\
\hline C4B & 0.50778 (19) & $-0.0046(2)$ & $0.58491(15)$ & $0.0438(5)$ & \\
\hline H4B & 0.513277 & -0.007863 & 0.642327 & $0.053 *$ & \\
\hline $\mathrm{O} 1 \mathrm{C}$ & $0.75142(13)$ & $0.4275(2)$ & $0.31558(11)$ & $0.0528(4)$ & \\
\hline $\mathrm{C} 1 \mathrm{C}$ & $0.7187(2)$ & $0.4691(3)$ & $0.24174(18)$ & $0.0558(6)$ & \\
\hline $\mathrm{H} 1 \mathrm{C}$ & 0.664285 & 0.529957 & 0.228484 & $0.067 *$ & \\
\hline $\mathrm{N} 1 \mathrm{C}$ & $0.75234(19)$ & $0.4370(3)$ & $0.18062(15)$ & $0.0567(6)$ & \\
\hline $\mathrm{C} 2 \mathrm{C}$ & $0.7109(3)$ & $0.4958(5)$ & $0.0954(2)$ & $0.0936(12)$ & \\
\hline $\mathrm{H} 2 \mathrm{CA}$ & 0.682464 & 0.422950 & 0.055091 & $0.140^{*}$ & \\
\hline $\mathrm{H} 2 \mathrm{CB}$ & 0.763583 & 0.541335 & 0.080424 & $0.140 *$ & \\
\hline $\mathrm{H} 2 \mathrm{CC}$ & 0.659234 & 0.561783 & 0.094316 & $0.140 *$ & \\
\hline $\mathrm{C} 3 \mathrm{C}$ & $0.8336(4)$ & $0.3417(6)$ & $0.1934(3)$ & 0.1189 (17) & \\
\hline $\mathrm{H} 3 \mathrm{CA}$ & 0.880182 & 0.351911 & 0.250325 & $0.178^{*}$ & \\
\hline $\mathrm{H} 3 \mathrm{CB}$ & 0.867587 & 0.360371 & 0.152930 & $0.178 *$ & \\
\hline $\mathrm{H} 3 \mathrm{CC}$ & 0.807666 & 0.248664 & 0.185574 & $0.178^{*}$ & \\
\hline O1D & $0.8014(9)$ & $0.3151(12)$ & $0.4826(6)$ & $0.0530(6)$ & $0.502(2)$ \\
\hline C1D & $0.8794(4)$ & $0.3625(6)$ & 0.5229 & $0.0571(10)$ & $0.502(2)$ \\
\hline H1D & 0.897601 & 0.443924 & 0.501510 & $0.069^{*}$ & $0.502(2)$ \\
\hline N1D & $0.9445(4)$ & $0.3160(7)$ & $0.5941(3)$ & $0.0713(9)$ & $0.502(2)$ \\
\hline C2D & $1.0427(5)$ & $0.3755(9)$ & $0.6322(5)$ & $0.101(2)$ & $0.502(2)$ \\
\hline
\end{tabular}




$\begin{array}{llllll}\text { H2DA } & 1.040810 & 0.440932 & 0.675326 & 0.151^{*} & 0.502(2) \\ \text { H2DB } & 1.062711 & 0.422124 & 0.589159 & 0.151^{*} & 0.502(2) \\ \text { H2DC } & 1.090317 & 0.303414 & 0.657648 & 0.151^{*} & 0.502(2) \\ \text { C3D } & 0.9207(6) & 0.1915(9) & 0.6338(5) & 0.109(2) & 0.502(2) \\ \text { H3DA } & 0.892358 & 0.122527 & 0.590807 & 0.164^{*} & 0.502(2) \\ \text { H3DB } & 0.872814 & 0.214163 & 0.661918 & 0.164^{*} & 0.502(2) \\ \text { H3DC } & 0.981193 & 0.155916 & 0.674938 & 0.164^{*} & 0.502(2) \\ \text { O1AD } & 0.7959(15) & 0.317(4) & 0.4743(6) & 0.0530(6) & 0.285(3) \\ \text { C1AD } & 0.8215(6) & 0.3181(12) & 0.5491(5) & 0.0587(12) & 0.285(3) \\ \text { H1AD } & 0.774908 & 0.353722 & 0.572603 & 0.070^{*} & 0.285(3) \\ \text { N1AD } & 0.9073(5) & 0.2759(10) & 0.6039(4) & 0.0713(9) & 0.285(3) \\ \text { C2AD } & 0.9272(8) & 0.2782(15) & 0.6944(4) & 0.085(3) & 0.285(3) \\ \text { H2DD } & 0.920908 & 0.371541 & 0.712322 & 0.127^{*} & 0.285(3) \\ \text { H2DE } & 0.994468 & 0.245201 & 0.722992 & 0.127^{*} & 0.285(3) \\ \text { H2DF } & 0.879667 & 0.219575 & 0.708430 & 0.127^{*} & 0.285(3) \\ \text { C3AD } & 0.9776(7) & 0.1978(14) & 0.5730(6) & 0.090(3) & 0.285(3) \\ \text { H3DD } & 0.941746 & 0.125546 & 0.535186 & 0.135^{*} & 0.285(3) \\ \text { H3DE } & 1.029120 & 0.157344 & 0.620566 & 0.135^{*} & 0.285(3) \\ \text { H3DF } & 1.008036 & 0.259050 & 0.542830 & 0.135^{*} & 0.285(3) \\ \text { O1BD } & 0.7929(14) & 0.315(4) & 0.4838(9) & 0.0530(6) & 0.213(3) \\ \text { C1BD } & 0.8318(6) & 0.3434(18) & 0.5559(6) & 0.0600(13) & 0.213(3) \\ \text { H1BD } & 0.792321 & 0.392423 & 0.581459 & 0.072^{*} & 0.213(3) \\ \text { N1BD } & 0.9240(5) & 0.3147(15) & 0.6053(5) & 0.0713(9) & 0.213(3) \\ \text { C2BD } & 0.9529(10) & 0.343(2) & 0.6968(5) & 0.087(3) & 0.213(3) \\ \text { H2DG } & 1.025064 & 0.340840 & 0.721607 & 0.131^{*} & 0.213(3) \\ \text { H2DH } & 0.924006 & 0.273742 & 0.723433 & 0.131^{*} & 0.213(3) \\ \text { H2DI } & 0.928462 & 0.432967 & 0.705483 & 0.131^{*} & 0.213(3) \\ \text { C3BD } & 1.0039(7) & 0.2844(19) & 0.5720(7) & 0.081(3) & 0.213(3) \\ \text { H3DG } & 0.976030 & 0.268049 & 0.511667 & 0.122^{*} & 0.213(3) \\ \text { H3DH } & 1.039618 & 0.203269 & 0.599686 & 0.122^{*} & 0.213(3) \\ \text { H3DI } & 1.049555 & 0.361718 & 0.582424 & 0.122^{*} & 0.213(3)\end{array}$

Atomic displacement parameters $\left(\AA^{2}\right)$

\begin{tabular}{lllllll}
\hline & $U^{11}$ & $U^{22}$ & $U^{33}$ & $U^{12}$ & $U^{13}$ & $U^{23}$ \\
\hline Ni1 & $0.0235(2)$ & $0.0262(2)$ & $0.0194(2)$ & $-0.00175(17)$ & $0.00091(16)$ & $0.00035(17)$ \\
Ni2 & $0.02733(18)$ & $0.0330(2)$ & $0.02504(18)$ & $0.00005(13)$ & $0.00201(13)$ & $-0.00149(14)$ \\
O1A & $0.0326(7)$ & $0.0499(9)$ & $0.0283(7)$ & $0.0057(7)$ & $0.0033(6)$ & $0.0066(7)$ \\
C1A & $0.0318(10)$ & $0.0330(10)$ & $0.0252(9)$ & $-0.0013(8)$ & $0.0023(8)$ & $-0.0015(8)$ \\
O2A & $0.0353(8)$ & $0.0580(10)$ & $0.0237(7)$ & $0.0064(7)$ & $0.0032(6)$ & $0.0076(7)$ \\
C2A & $0.0322(10)$ & $0.0405(11)$ & $0.0268(9)$ & $0.0038(9)$ & $0.0024(8)$ & $0.0043(9)$ \\
O3A & $0.0362(7)$ & $0.0418(8)$ & $0.0330(7)$ & $0.0109(6)$ & $0.0074(6)$ & $0.0090(6)$ \\
C3A & $0.0301(10)$ & $0.0671(16)$ & $0.0363(11)$ & $0.0127(10)$ & $0.0075(9)$ & $0.0136(11)$ \\
O4A & $0.0290(7)$ & $0.0351(8)$ & $0.0463(8)$ & $0.0070(6)$ & $0.0058(6)$ & $0.0090(7)$ \\
C3B & $0.0567(14)$ & $0.0279(11)$ & $0.0424(12)$ & $-0.0084(10)$ & $0.0115(10)$ & $-0.0070(9)$ \\
C5A & $0.0305(10)$ & $0.0384(11)$ & $0.0276(9)$ & $0.0065(8)$ & $0.0022(8)$ & $0.0034(8)$ \\
C6A & $0.0314(11)$ & $0.086(2)$ & $0.0464(13)$ & $0.0138(12)$ & $0.0116(10)$ & $0.0248(14)$ \\
C7A & $0.0384(12)$ & $0.090(2)$ & $0.0422(13)$ & $0.0134(13)$ & $0.0139(10)$ & $0.0298(14)$
\end{tabular}




\begin{tabular}{|c|c|c|c|c|c|c|}
\hline C8A & $0.0309(10)$ & $0.0291(10)$ & $0.0289(9)$ & $0.0042(8)$ & $0.0023(8)$ & $0.0002(8)$ \\
\hline $\mathrm{C} 4 \mathrm{~A}$ & $0.0366(11)$ & $0.0638(16)$ & 0.0319 (11) & $0.0110(11)$ & $0.0098(9)$ & $0.0157(11)$ \\
\hline O1B & $0.0556(9)$ & $0.0363(8)$ & $0.0430(9)$ & $-0.0060(7)$ & $0.0174(7)$ & $-0.0057(7)$ \\
\hline C1B & $0.0325(10)$ & $0.0291(10)$ & $0.0377(11)$ & $0.0017(8)$ & $0.0044(8)$ & $0.0000(9)$ \\
\hline $\mathrm{O} 2 \mathrm{~B}$ & $0.0324(7)$ & $0.0250(7)$ & $0.0365(7)$ & $-0.0010(6)$ & $0.0074(6)$ & $-0.0015(6)$ \\
\hline C2B & $0.0376(11)$ & $0.0252(10)$ & $0.0438(11)$ & $0.0010(8)$ & 0.0099 (9) & $-0.0004(9)$ \\
\hline C4B & $0.0583(14)$ & $0.0333(12)$ & $0.0385(11)$ & $-0.0052(10)$ & $0.0140(10)$ & $-0.0034(10)$ \\
\hline $\mathrm{O} 1 \mathrm{C}$ & $0.0464(9)$ & $0.0731(12)$ & $0.0419(9)$ & $0.0095(8)$ & $0.0188(7)$ & $0.0067(9)$ \\
\hline $\mathrm{C} 1 \mathrm{C}$ & $0.0560(14)$ & $0.0654(16)$ & 0.0487 (13) & $0.0090(12)$ & $0.0208(11)$ & $0.0019(12)$ \\
\hline $\mathrm{N} 1 \mathrm{C}$ & $0.0714(14)$ & $0.0577(13)$ & $0.0486(12)$ & $-0.0040(11)$ & $0.0301(11)$ & $-0.0011(10)$ \\
\hline $\mathrm{C} 2 \mathrm{C}$ & $0.110(3)$ & $0.120(3)$ & $0.0534(18)$ & $-0.020(3)$ & 0.0299 (19) & $0.011(2)$ \\
\hline $\mathrm{C} 3 \mathrm{C}$ & 0.149 (4) & $0.127(4)$ & $0.109(3)$ & $0.053(3)$ & $0.080(3)$ & $0.012(3)$ \\
\hline O1D & $0.0397(12)$ & $0.0572(10)$ & $0.0484(10)$ & $0.0110(9)$ & $-0.0040(9)$ & $0.0014(14)$ \\
\hline C1D & $0.0406(16)$ & $0.0618(18)$ & $0.0543(17)$ & $0.0079(16)$ & $-0.0040(15)$ & $0.0058(17)$ \\
\hline N1D & $0.0537(15)$ & $0.0745(15)$ & 0.0625 (13) & 0.0094 (13) & $-0.0124(12)$ & $0.0064(13)$ \\
\hline $\mathrm{C} 2 \mathrm{D}$ & $0.067(3)$ & $0.104(4)$ & $0.097(4)$ & $-0.001(3)$ & $-0.021(3)$ & -0.001 \\
\hline C3D & 0.094 (4) & $0.106(4)$ & 0.093 (4) & -0.003 & $-0.015(3)$ & $0.022(3)$ \\
\hline O1AD & $0.0397(12)$ & $0.0572(10)$ & $0.0484(10)$ & $0.0110(9)$ & $-0.0040(9)$ & $0.0014(14)$ \\
\hline $\mathrm{C} 1 \mathrm{AD}$ & 0.0454 (19) & $0.065(2)$ & $0.0521(19)$ & $0.0110(18)$ & $-0.0028(18)$ & $0.004(2)$ \\
\hline N1AD & $0.0537(15)$ & $0.0745(15)$ & $0.0625(13)$ & $0.0094(13)$ & $-0.0124(12)$ & $0.0064(13)$ \\
\hline C2AD & $0.077(4)$ & $0.083(4)$ & $0.070(4)$ & $0.005(4)$ & $-0.009(4)$ & $0.009(4)$ \\
\hline C3AD & $0.065(4)$ & $0.095(4)$ & $0.083(4)$ & 0.015 & -0.011 & $0.004(4)$ \\
\hline O1BD & $0.0397(12)$ & $0.0572(10)$ & $0.0484(10)$ & $0.0110(9)$ & $-0.0040(9)$ & $0.0014(14)$ \\
\hline C1BD & $0.046(2)$ & $0.065(2)$ & $0.053(2)$ & 0.0109 (19) & $-0.0046(19)$ & $0.004(2)$ \\
\hline N1BD & $0.0537(15)$ & $0.0745(15)$ & $0.0625(13)$ & 0.0094 (13) & $-0.0124(12)$ & $0.0064(13)$ \\
\hline C2BD & $0.071(4)$ & $0.092(4)$ & $0.071(4)$ & $0.001(4)$ & $-0.013(4)$ & 0.007 (4) \\
\hline C3BD & $0.061(4)$ & $0.087(4)$ & $0.074(4)$ & 0.007 (4) & -0.008 (4) & $0.003(4)$ \\
\hline
\end{tabular}

Geometric parameters $\left(\AA,{ }^{\circ}\right)$

\begin{tabular}{llll}
\hline $\mathrm{Ni} 1-\mathrm{O} 2 \mathrm{~A}^{\mathrm{i}}$ & $2.0205(14)$ & $\mathrm{N} 1 \mathrm{C}-\mathrm{C} 3 \mathrm{C}$ & $1.424(5)$ \\
$\mathrm{Ni1}-\mathrm{O} 2 \mathrm{~A}$ & $2.0206(14)$ & $\mathrm{N} 1 \mathrm{C}-\mathrm{C} 2 \mathrm{C}$ & $1.454(4)$ \\
$\mathrm{Ni1}-\mathrm{O} 4 \mathrm{~A}^{\mathrm{ii}}$ & $2.0246(14)$ & $\mathrm{C} 2 \mathrm{C}-\mathrm{H} 2 \mathrm{CA}$ & 0.9600 \\
$\mathrm{Ni1}-\mathrm{O} 4 \mathrm{~A}^{\mathrm{iii}}$ & $2.0246(14)$ & $\mathrm{C} 2 \mathrm{C}-\mathrm{H} 2 \mathrm{CB}$ & 0.9600 \\
$\mathrm{Ni1}-\mathrm{O} 2 \mathrm{~B}^{\mathrm{i}}$ & $2.0868(14)$ & $\mathrm{C} 2 \mathrm{C}-\mathrm{H} 2 \mathrm{CC}$ & 0.9600 \\
$\mathrm{Ni1}-\mathrm{O} 2 \mathrm{~B}$ & $2.0868(14)$ & $\mathrm{C} 3 \mathrm{C}-\mathrm{H} 3 \mathrm{CA}$ & 0.9600 \\
$\mathrm{Ni2}-\mathrm{O} 3 \mathrm{~A}^{\mathrm{ii}}$ & $2.0090(15)$ & $\mathrm{C} 3 \mathrm{C}-\mathrm{H} 3 \mathrm{CB}$ & 0.9600 \\
$\mathrm{Ni2}-\mathrm{O} 1 \mathrm{~A}$ & $2.0184(15)$ & $\mathrm{C} 3 \mathrm{C}-\mathrm{H} 3 \mathrm{CC}$ & 0.9600 \\
$\mathrm{Ni2}-\mathrm{O} 1 \mathrm{C}$ & $2.0399(17)$ & $\mathrm{O} 1 \mathrm{D}-\mathrm{C} 1 \mathrm{D}$ & $1.171(10)$ \\
$\mathrm{Ni2}-\mathrm{O} 1 \mathrm{AD}$ & $2.042(12)$ & $\mathrm{C} 1 \mathrm{D}-\mathrm{N} 1 \mathrm{D}$ & $1.311(4)$ \\
$\mathrm{Ni2}-\mathrm{O} 2 \mathrm{~B}$ & $2.0791(15)$ & $\mathrm{C} 1 \mathrm{D}-\mathrm{H} 1 \mathrm{D}$ & 0.9300 \\
$\mathrm{Ni2}-\mathrm{O} 1 \mathrm{BD}$ & $2.081(19)$ & $\mathrm{N} 1 \mathrm{D}-\mathrm{C} 2 \mathrm{D}$ & $1.432(7)$ \\
$\mathrm{Ni2}-\mathrm{O} 1 \mathrm{D}$ & $2.146(6)$ & $\mathrm{N} 1 \mathrm{D}-\mathrm{C} 3 \mathrm{D}$ & $1.460(8)$ \\
$\mathrm{Ni2}-\mathrm{O} 1 \mathrm{~B}$ & $2.1853(16)$ & $\mathrm{C} 2 \mathrm{D}-\mathrm{H} 2 \mathrm{DA}$ & 0.9600 \\
$\mathrm{Ni2}-\mathrm{C} 1 \mathrm{~B}$ & $2.465(2)$ & $\mathrm{C} 2 \mathrm{D}-\mathrm{H} 2 \mathrm{DB}$ & 0.9600 \\
$\mathrm{O} 1 \mathrm{~A}-\mathrm{C} 1 \mathrm{~A}$ & $1.255(3)$ & $\mathrm{C} 2 \mathrm{D}-\mathrm{H} 2 \mathrm{DC}$ & 0.9600 \\
$\mathrm{C} 1 \mathrm{~A}-\mathrm{O} 2 \mathrm{~A}$ & $1.237(3)$ & $\mathrm{C} 3 \mathrm{D}-\mathrm{H} 3 \mathrm{DA}$ & 0.9600 \\
$\mathrm{C} 1 \mathrm{~A}-\mathrm{C} 2 \mathrm{~A}$ & $1.514(3)$ & $\mathrm{C} 3 \mathrm{D}-\mathrm{H} 3 \mathrm{DB}$ & 0.9600
\end{tabular}




\begin{tabular}{|c|c|c|c|}
\hline $\mathrm{C} 2 \mathrm{~A}-\mathrm{C} 3 \mathrm{~A}$ & $1.379(3)$ & $\mathrm{C} 3 \mathrm{D}-\mathrm{H} 3 \mathrm{DC}$ & 0.9600 \\
\hline $\mathrm{C} 2 \mathrm{~A}-\mathrm{C} 7 \mathrm{~A}$ & $1.384(3)$ & $\mathrm{O} 1 \mathrm{AD}-\mathrm{C} 1 \mathrm{AD}$ & $1.171(10)$ \\
\hline $\mathrm{O} 3 \mathrm{~A}-\mathrm{C} 8 \mathrm{~A}$ & $1.266(3)$ & $\mathrm{C} 1 \mathrm{AD}-\mathrm{N} 1 \mathrm{AD}$ & $1.312(4)$ \\
\hline $\mathrm{C} 3 \mathrm{~A}-\mathrm{C} 4 \mathrm{~A}$ & $1.383(3)$ & $\mathrm{C} 1 \mathrm{AD}-\mathrm{H} 1 \mathrm{AD}$ & 0.9300 \\
\hline $\mathrm{C} 3 \mathrm{~A}-\mathrm{H} 3 \mathrm{~A}$ & 0.9300 & $\mathrm{~N} 1 \mathrm{AD}-\mathrm{C} 2 \mathrm{AD}$ & $1.433(7)$ \\
\hline $\mathrm{O} 4 \mathrm{~A}-\mathrm{C} 8 \mathrm{~A}$ & $1.237(2)$ & $\mathrm{N} 1 \mathrm{AD}-\mathrm{C} 3 \mathrm{AD}$ & $1.461(8)$ \\
\hline $\mathrm{C} 3 \mathrm{~B}-\mathrm{C} 4 \mathrm{~B}$ & $1.381(3)$ & $\mathrm{C} 2 \mathrm{AD}-\mathrm{H} 2 \mathrm{DD}$ & 0.9600 \\
\hline $\mathrm{C} 3 \mathrm{~B}-\mathrm{C} 2 \mathrm{~B}$ & $1.381(3)$ & $\mathrm{C} 2 \mathrm{AD}-\mathrm{H} 2 \mathrm{DE}$ & 0.9600 \\
\hline $\mathrm{C} 3 \mathrm{~B}-\mathrm{H} 3 \mathrm{~B}$ & 0.9300 & $\mathrm{C} 2 \mathrm{AD}-\mathrm{H} 2 \mathrm{DF}$ & 0.9600 \\
\hline $\mathrm{C} 5 \mathrm{~A}-\mathrm{C} 6 \mathrm{~A}$ & $1.378(3)$ & $\mathrm{C} 3 \mathrm{AD}-\mathrm{H} 3 \mathrm{DD}$ & 0.9600 \\
\hline $\mathrm{C} 5 \mathrm{~A}-\mathrm{C} 4 \mathrm{~A}$ & $1.380(3)$ & $\mathrm{C} 3 \mathrm{AD}-\mathrm{H} 3 \mathrm{DE}$ & 0.9600 \\
\hline $\mathrm{C} 5 \mathrm{~A}-\mathrm{C} 8 \mathrm{~A}$ & $1.506(3)$ & $\mathrm{C} 3 \mathrm{AD}-\mathrm{H} 3 \mathrm{DF}$ & 0.9600 \\
\hline $\mathrm{C} 6 \mathrm{~A}-\mathrm{C} 7 \mathrm{~A}$ & $1.379(3)$ & $\mathrm{O} 1 \mathrm{BD}-\mathrm{C} 1 \mathrm{BD}$ & $1.171(10)$ \\
\hline C6A-H6A & 0.9300 & $\mathrm{C} 1 \mathrm{BD}-\mathrm{N} 1 \mathrm{BD}$ & $1.312(4)$ \\
\hline $\mathrm{C} 7 \mathrm{~A}-\mathrm{H} 7 \mathrm{~A}$ & 0.9300 & $\mathrm{C} 1 \mathrm{BD}-\mathrm{H} 1 \mathrm{BD}$ & 0.9300 \\
\hline $\mathrm{C} 4 \mathrm{~A}-\mathrm{H} 4 \mathrm{~A}$ & 0.9300 & $\mathrm{~N} 1 \mathrm{BD}-\mathrm{C} 3 \mathrm{BD}$ & $1.433(7)$ \\
\hline $\mathrm{O} 1 \mathrm{~B}-\mathrm{C} 1 \mathrm{~B}$ & $1.244(3)$ & $\mathrm{N} 1 \mathrm{BD}-\mathrm{C} 2 \mathrm{BD}$ & $1.461(8)$ \\
\hline $\mathrm{C} 1 \mathrm{~B}-\mathrm{O} 2 \mathrm{~B}$ & $1.284(3)$ & $\mathrm{C} 2 \mathrm{BD}-\mathrm{H} 2 \mathrm{DG}$ & 0.9600 \\
\hline $\mathrm{C} 1 \mathrm{~B}-\mathrm{C} 2 \mathrm{~B}$ & $1.491(3)$ & $\mathrm{C} 2 \mathrm{BD}-\mathrm{H} 2 \mathrm{DH}$ & 0.9600 \\
\hline $\mathrm{C} 2 \mathrm{~B}-\mathrm{C} 4 \mathrm{~B}^{\mathrm{iv}}$ & $1.389(3)$ & $\mathrm{C} 2 \mathrm{BD}-\mathrm{H} 2 \mathrm{DI}$ & 0.9600 \\
\hline C4B-H4B & 0.9300 & $\mathrm{C} 3 \mathrm{BD}-\mathrm{H} 3 \mathrm{DG}$ & 0.9600 \\
\hline $\mathrm{O} 1 \mathrm{C}-\mathrm{C} 1 \mathrm{C}$ & $1.225(3)$ & $\mathrm{C} 3 \mathrm{BD}-\mathrm{H} 3 \mathrm{DH}$ & 0.9600 \\
\hline $\mathrm{C} 1 \mathrm{C}-\mathrm{N} 1 \mathrm{C}$ & $1.288(4)$ & C3BD-H3DI & 0.9600 \\
\hline $\mathrm{C} 1 \mathrm{C}-\mathrm{H} 1 \mathrm{C}$ & 0.9300 & & \\
\hline $\mathrm{O} 2 \mathrm{~A}^{\mathrm{i}}-\mathrm{Ni} 1-\mathrm{O} 2 \mathrm{~A}$ & 180.0 & $\mathrm{C} 1 \mathrm{~B}-\mathrm{O} 2 \mathrm{~B}-\mathrm{Ni} 2$ & $91.14(13)$ \\
\hline $\mathrm{O} 2 \mathrm{~A}^{\mathrm{i}}-\mathrm{Ni} 1-\mathrm{O} 4 \mathrm{~A}^{\mathrm{ii}}$ & $85.85(7)$ & $\mathrm{C} 1 \mathrm{~B}-\mathrm{O} 2 \mathrm{~B}-\mathrm{Ni} 1$ & $131.39(13)$ \\
\hline $\mathrm{O} 2 \mathrm{~A}-\mathrm{Ni} 1-\mathrm{O} 4 \mathrm{~A}^{\mathrm{ii}}$ & $94.15(7)$ & $\mathrm{Ni} 2-\mathrm{O} 2 \mathrm{~B}-\mathrm{Ni} 1$ & $111.40(6)$ \\
\hline $\mathrm{O} 2 \mathrm{~A}^{\mathrm{i}}-\mathrm{Ni} 1-\mathrm{O} 4 \mathrm{~A}^{\mathrm{iii}}$ & $94.15(7)$ & $\mathrm{C} 3 \mathrm{~B}-\mathrm{C} 2 \mathrm{~B}-\mathrm{C} 4 \mathrm{~B}^{\mathrm{iv}}$ & $119.4(2)$ \\
\hline $\mathrm{O} 2 \mathrm{~A}-\mathrm{Ni} 1-\mathrm{O} 4 \mathrm{~A}^{\mathrm{iii}}$ & $85.85(7)$ & $\mathrm{C} 3 \mathrm{~B}-\mathrm{C} 2 \mathrm{~B}-\mathrm{C} 1 \mathrm{~B}$ & $120.3(2)$ \\
\hline $\mathrm{O} 4 \mathrm{~A}^{\mathrm{ii}}-\mathrm{Ni} 1-\mathrm{O} 4 \mathrm{~A}^{\mathrm{iii}}$ & $180.00(12)$ & $\mathrm{C} 4 \mathrm{~B}^{\mathrm{iv}}-\mathrm{C} 2 \mathrm{~B}-\mathrm{C} 1 \mathrm{~B}$ & $120.3(2)$ \\
\hline $\mathrm{O} 2 \mathrm{~A}^{\mathrm{i}}-\mathrm{Ni} 1-\mathrm{O} 2 \mathrm{~B}^{\mathrm{i}}$ & $91.60(6)$ & $\mathrm{C} 3 \mathrm{~B}-\mathrm{C} 4 \mathrm{~B}-\mathrm{C} 2 \mathrm{~B}^{\mathrm{iv}}$ & $120.6(2)$ \\
\hline $\mathrm{O} 2 \mathrm{~A}-\mathrm{Ni} 1-\mathrm{O} 2 \mathrm{~B}^{\mathrm{i}}$ & $88.40(6)$ & $\mathrm{C} 3 \mathrm{~B}-\mathrm{C} 4 \mathrm{~B}-\mathrm{H} 4 \mathrm{~B}$ & 119.7 \\
\hline $\mathrm{O} 4 \mathrm{~A}^{\mathrm{ii}}-\mathrm{Ni} 1-\mathrm{O} 2 \mathrm{~B}^{\mathrm{i}}$ & $90.75(6)$ & $\mathrm{C} 2 \mathrm{~B}^{\mathrm{iv}}-\mathrm{C} 4 \mathrm{~B}-\mathrm{H} 4 \mathrm{~B}$ & 119.7 \\
\hline 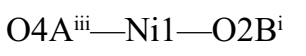 & $89.25(6)$ & $\mathrm{C} 1 \mathrm{C}-\mathrm{O} 1 \mathrm{C}-\mathrm{Ni} 2$ & $125.55(17)$ \\
\hline $\mathrm{O} 2 \mathrm{~A}^{\mathrm{i}}-\mathrm{Ni1}-\mathrm{O} 2 \mathrm{~B}$ & $88.40(6)$ & $\mathrm{O} 1 \mathrm{C}-\mathrm{C} 1 \mathrm{C}-\mathrm{N} 1 \mathrm{C}$ & $126.5(3)$ \\
\hline $\mathrm{O} 2 \mathrm{~A}-\mathrm{Ni} 1-\mathrm{O} 2 \mathrm{~B}$ & $91.60(6)$ & $\mathrm{O} 1 \mathrm{C}-\mathrm{C} 1 \mathrm{C}-\mathrm{H} 1 \mathrm{C}$ & 116.8 \\
\hline 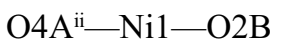 & $89.25(6)$ & $\mathrm{N} 1 \mathrm{C}-\mathrm{C} 1 \mathrm{C}-\mathrm{H} 1 \mathrm{C}$ & 116.8 \\
\hline 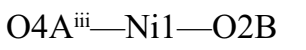 & $90.75(6)$ & $\mathrm{C} 1 \mathrm{C}-\mathrm{N} 1 \mathrm{C}-\mathrm{C} 3 \mathrm{C}$ & $121.3(3)$ \\
\hline $\mathrm{O} 2 \mathrm{~B}^{\mathrm{i}}-\mathrm{Ni} 1-\mathrm{O} 2 \mathrm{~B}$ & $180.00(5)$ & $\mathrm{C} 1 \mathrm{C}-\mathrm{N} 1 \mathrm{C}-\mathrm{C} 2 \mathrm{C}$ & $122.6(3)$ \\
\hline $\mathrm{O} 3 \mathrm{~A}^{\mathrm{ii}}-\mathrm{Ni2}-\mathrm{O} 1 \mathrm{~A}$ & $97.00(7)$ & $\mathrm{C} 3 \mathrm{C}-\mathrm{N} 1 \mathrm{C}-\mathrm{C} 2 \mathrm{C}$ & $116.1(3)$ \\
\hline $\mathrm{O} 3 \mathrm{~A}^{\mathrm{ii}}-\mathrm{Ni2}-\mathrm{O} 1 \mathrm{C}$ & $99.13(7)$ & $\mathrm{N} 1 \mathrm{C}-\mathrm{C} 2 \mathrm{C}-\mathrm{H} 2 \mathrm{CA}$ & 109.5 \\
\hline $\mathrm{O} 1 \mathrm{~A}-\mathrm{Ni2}-\mathrm{O} 1 \mathrm{C}$ & $88.63(7)$ & $\mathrm{N} 1 \mathrm{C}-\mathrm{C} 2 \mathrm{C}-\mathrm{H} 2 \mathrm{CB}$ & 109.5 \\
\hline $\mathrm{O} 3 \mathrm{~A}^{\mathrm{ii}}-\mathrm{Ni} 2-\mathrm{O} 1 \mathrm{AD}$ & $87.1(9)$ & $\mathrm{H} 2 \mathrm{CA}-\mathrm{C} 2 \mathrm{C}-\mathrm{H} 2 \mathrm{CB}$ & 109.5 \\
\hline $\mathrm{O} 1 \mathrm{~A}-\mathrm{Ni2}-\mathrm{O} 1 \mathrm{AD}$ & $171.6(5)$ & $\mathrm{N} 1 \mathrm{C}-\mathrm{C} 2 \mathrm{C}-\mathrm{H} 2 \mathrm{CC}$ & 109.5 \\
\hline $\mathrm{O} 1 \mathrm{C}-\mathrm{Ni} 2-\mathrm{O} 1 \mathrm{AD}$ & $83.4(7)$ & $\mathrm{H} 2 \mathrm{CA}-\mathrm{C} 2 \mathrm{C}-\mathrm{H} 2 \mathrm{CC}$ & 109.5 \\
\hline $\mathrm{O} 3 \mathrm{~A}^{\mathrm{ii}}-\mathrm{Ni} 2-\mathrm{O} 2 \mathrm{~B}$ & $99.18(6)$ & $\mathrm{H} 2 \mathrm{CB}-\mathrm{C} 2 \mathrm{C}-\mathrm{H} 2 \mathrm{CC}$ & 109.5 \\
\hline $\mathrm{O} 1 \mathrm{~A}-\mathrm{Ni} 2-\mathrm{O} 2 \mathrm{~B}$ & $99.20(6)$ & $\mathrm{N} 1 \mathrm{C}-\mathrm{C} 3 \mathrm{C}-\mathrm{H} 3 \mathrm{CA}$ & 109.5 \\
\hline
\end{tabular}




\begin{tabular}{|c|c|}
\hline $\mathrm{O} 1 \mathrm{C}-\mathrm{Ni} 2-\mathrm{O} 2 \mathrm{~B}$ & $159.02(7)$ \\
\hline $\mathrm{O} 1 \mathrm{AD}-\mathrm{Ni} 2-\mathrm{O} 2 \mathrm{~B}$ & $87.4(9)$ \\
\hline $\mathrm{O} 3 \mathrm{~A}^{\mathrm{ii}}-\mathrm{Ni} 2-\mathrm{O} 1 \mathrm{BD}$ & $85.9(9)$ \\
\hline $\mathrm{O} 1 \mathrm{~A}-\mathrm{Ni} 2-\mathrm{O} 1 \mathrm{BD}$ & $176.0(6)$ \\
\hline $\mathrm{O} 1 \mathrm{C}-\mathrm{Ni2}-\mathrm{O} 1 \mathrm{BD}$ & $88.2(7)$ \\
\hline $\mathrm{O} 2 \mathrm{~B}-\mathrm{Ni} 2-\mathrm{O} 1 \mathrm{BD}$ & $83.0(9)$ \\
\hline $\mathrm{O} 3 \mathrm{~A}^{\mathrm{ii}}-\mathrm{Ni} 2-\mathrm{O} 1 \mathrm{D}$ & $85.5(3)$ \\
\hline $\mathrm{O} 1 \mathrm{~A}-\mathrm{Ni2}-\mathrm{O} 1 \mathrm{D}$ & $173.8(4)$ \\
\hline $\mathrm{O} 1 \mathrm{C}-\mathrm{Ni2}-\mathrm{O} 1 \mathrm{D}$ & $85.3(5)$ \\
\hline $\mathrm{O} 2 \mathrm{~B}-\mathrm{Ni2}-\mathrm{O} 1 \mathrm{D}$ & $86.0(5)$ \\
\hline $\mathrm{O} 3 \mathrm{~A}^{\mathrm{ii}}-\mathrm{Ni2}-\mathrm{O} 1 \mathrm{~B}$ & $159.66(6)$ \\
\hline $\mathrm{O} 1 \mathrm{~A}-\mathrm{Ni2}-\mathrm{O} 1 \mathrm{~B}$ & $92.73(7)$ \\
\hline $\mathrm{O} 1 \mathrm{C}-\mathrm{Ni2}-\mathrm{O} 1 \mathrm{~B}$ & $98.91(7)$ \\
\hline $\mathrm{O} 1 \mathrm{AD}-\mathrm{Ni2}-\mathrm{O} 1 \mathrm{~B}$ & $85.7(10)$ \\
\hline $\mathrm{O} 2 \mathrm{~B}-\mathrm{Ni2}-\mathrm{O} 1 \mathrm{~B}$ & $61.52(6)$ \\
\hline $\mathrm{O} 1 \mathrm{BD}-\mathrm{Ni} 2-\mathrm{O} 1 \mathrm{~B}$ & $85.4(10)$ \\
\hline O1D-Ni2-O1B & $86.7(4)$ \\
\hline $\mathrm{O} 3 \mathrm{~A}^{\mathrm{ii}}-\mathrm{Ni2}-\mathrm{C} 1 \mathrm{~B}$ & $129.86(7)$ \\
\hline $\mathrm{O} 1 \mathrm{~A}-\mathrm{Ni2}-\mathrm{C} 1 \mathrm{~B}$ & $99.00(7)$ \\
\hline $\mathrm{O} 1 \mathrm{C}-\mathrm{Ni} 2-\mathrm{C} 1 \mathrm{~B}$ & $128.35(8)$ \\
\hline $\mathrm{O} 1 \mathrm{AD}-\mathrm{Ni2}-\mathrm{C} 1 \mathrm{~B}$ & $83.8(11)$ \\
\hline $\mathrm{O} 2 \mathrm{~B}-\mathrm{Ni2}-\mathrm{C} 1 \mathrm{~B}$ & $31.38(6)$ \\
\hline $\mathrm{O} 1 \mathrm{BD}-\mathrm{Ni} 2-\mathrm{C} 1 \mathrm{~B}$ & $81.1(11)$ \\
\hline $\mathrm{O} 1 \mathrm{D}-\mathrm{Ni} 2-\mathrm{C} 1 \mathrm{~B}$ & $83.6(5)$ \\
\hline $\mathrm{O} 1 \mathrm{~B}-\mathrm{Ni2}-\mathrm{C} 1 \mathrm{~B}$ & $30.28(7)$ \\
\hline $\mathrm{C} 1 \mathrm{~A}-\mathrm{O} 1 \mathrm{~A}-\mathrm{Ni2}$ & $129.89(14)$ \\
\hline $\mathrm{O} 2 \mathrm{~A}-\mathrm{C} 1 \mathrm{~A}-\mathrm{O} 1 \mathrm{~A}$ & $127.48(18)$ \\
\hline $\mathrm{O} 2 \mathrm{~A}-\mathrm{C} 1 \mathrm{~A}-\mathrm{C} 2 \mathrm{~A}$ & $115.67(18)$ \\
\hline $\mathrm{O} 1 \mathrm{~A}-\mathrm{C} 1 \mathrm{~A}-\mathrm{C} 2 \mathrm{~A}$ & $116.84(18)$ \\
\hline $\mathrm{C} 1 \mathrm{~A}-\mathrm{O} 2 \mathrm{~A}-\mathrm{Ni1}$ & $135.62(14)$ \\
\hline $\mathrm{C} 3 \mathrm{~A}-\mathrm{C} 2 \mathrm{~A}-\mathrm{C} 7 \mathrm{~A}$ & $118.32(19)$ \\
\hline $\mathrm{C} 3 \mathrm{~A}-\mathrm{C} 2 \mathrm{~A}-\mathrm{C} 1 \mathrm{~A}$ & $122.19(19)$ \\
\hline $\mathrm{C} 7 \mathrm{~A}-\mathrm{C} 2 \mathrm{~A}-\mathrm{C} 1 \mathrm{~A}$ & $119.46(19)$ \\
\hline $\mathrm{C} 8 \mathrm{~A}-\mathrm{O} 3 \mathrm{~A}-\mathrm{Ni} 2^{\mathrm{v}}$ & $121.68(13)$ \\
\hline $\mathrm{C} 2 \mathrm{~A}-\mathrm{C} 3 \mathrm{~A}-\mathrm{C} 4 \mathrm{~A}$ & $120.9(2)$ \\
\hline $\mathrm{C} 2 \mathrm{~A}-\mathrm{C} 3 \mathrm{~A}-\mathrm{H} 3 \mathrm{~A}$ & 119.5 \\
\hline $\mathrm{C} 4 \mathrm{~A}-\mathrm{C} 3 \mathrm{~A}-\mathrm{H} 3 \mathrm{~A}$ & 119.5 \\
\hline $\mathrm{C} 8 \mathrm{~A}-\mathrm{O} 4 \mathrm{~A}-\mathrm{Ni1}{ }^{\mathrm{vi}}$ & $139.74(14)$ \\
\hline $\mathrm{C} 4 \mathrm{~B}-\mathrm{C} 3 \mathrm{~B}-\mathrm{C} 2 \mathrm{~B}$ & $120.0(2)$ \\
\hline $\mathrm{C} 4 \mathrm{~B}-\mathrm{C} 3 \mathrm{~B}-\mathrm{H} 3 \mathrm{~B}$ & 120.0 \\
\hline $\mathrm{C} 2 \mathrm{~B}-\mathrm{C} 3 \mathrm{~B}-\mathrm{H} 3 \mathrm{~B}$ & 120.0 \\
\hline $\mathrm{C} 6 \mathrm{~A}-\mathrm{C} 5 \mathrm{~A}-\mathrm{C} 4 \mathrm{~A}$ & $118.62(19)$ \\
\hline $\mathrm{C} 6 \mathrm{~A}-\mathrm{C} 5 \mathrm{~A}-\mathrm{C} 8 \mathrm{~A}$ & $119.45(19)$ \\
\hline $\mathrm{C} 4 \mathrm{~A}-\mathrm{C} 5 \mathrm{~A}-\mathrm{C} 8 \mathrm{~A}$ & $121.84(19)$ \\
\hline $\mathrm{C} 5 \mathrm{~A}-\mathrm{C} 6 \mathrm{~A}-\mathrm{C} 7 \mathrm{~A}$ & $120.8(2)$ \\
\hline $\mathrm{C} 5 \mathrm{~A}-\mathrm{C} 6 \mathrm{~A}-\mathrm{H} 6 \mathrm{~A}$ & 119.6 \\
\hline $\mathrm{C} 7 \mathrm{~A}-\mathrm{C} 6 \mathrm{~A}-\mathrm{H} 6 \mathrm{~A}$ & 119.6 \\
\hline $\mathrm{C} 6 \mathrm{~A}-\mathrm{C} 7 \mathrm{~A}-\mathrm{C} 2 \mathrm{~A}$ & $120.8(2)$ \\
\hline
\end{tabular}

\begin{tabular}{|c|c|}
\hline $\mathrm{N} 1 \mathrm{C}-\mathrm{C} 3 \mathrm{C}-\mathrm{H} 3 \mathrm{CB}$ & 109.5 \\
\hline $\mathrm{H} 3 \mathrm{CA}-\mathrm{C} 3 \mathrm{C}-\mathrm{H} 3 \mathrm{CB}$ & 109.5 \\
\hline $\mathrm{N} 1 \mathrm{C}-\mathrm{C} 3 \mathrm{C}-\mathrm{H} 3 \mathrm{CC}$ & 109.5 \\
\hline $\mathrm{H} 3 \mathrm{CA}-\mathrm{C} 3 \mathrm{C}-\mathrm{H} 3 \mathrm{CC}$ & 109.5 \\
\hline $\mathrm{H} 3 \mathrm{CB}-\mathrm{C} 3 \mathrm{C}-\mathrm{H} 3 \mathrm{CC}$ & 109.5 \\
\hline $\mathrm{C} 1 \mathrm{D}-\mathrm{O} 1 \mathrm{D}-\mathrm{Ni} 2$ & $126.2(8)$ \\
\hline $\mathrm{O} 1 \mathrm{D}-\mathrm{C} 1 \mathrm{D}-\mathrm{N} 1 \mathrm{D}$ & $128.5(7)$ \\
\hline $\mathrm{O} 1 \mathrm{D}-\mathrm{C} 1 \mathrm{D}-\mathrm{H} 1 \mathrm{D}$ & 115.7 \\
\hline $\mathrm{N} 1 \mathrm{D}-\mathrm{C} 1 \mathrm{D}-\mathrm{H} 1 \mathrm{D}$ & 115.7 \\
\hline $\mathrm{C} 1 \mathrm{D}-\mathrm{N} 1 \mathrm{D}-\mathrm{C} 2 \mathrm{D}$ & $123.0(5)$ \\
\hline $\mathrm{C} 1 \mathrm{D}-\mathrm{N} 1 \mathrm{D}-\mathrm{C} 3 \mathrm{D}$ & $119.5(5)$ \\
\hline $\mathrm{C} 2 \mathrm{D}-\mathrm{N} 1 \mathrm{D}-\mathrm{C} 3 \mathrm{D}$ & $117.4(4)$ \\
\hline $\mathrm{N} 1 \mathrm{D}-\mathrm{C} 2 \mathrm{D}-\mathrm{H} 2 \mathrm{DA}$ & 109.5 \\
\hline $\mathrm{N} 1 \mathrm{D}-\mathrm{C} 2 \mathrm{D}-\mathrm{H} 2 \mathrm{DB}$ & 109.5 \\
\hline $\mathrm{H} 2 \mathrm{DA}-\mathrm{C} 2 \mathrm{D}-\mathrm{H} 2 \mathrm{DB}$ & 109.5 \\
\hline $\mathrm{N} 1 \mathrm{D}-\mathrm{C} 2 \mathrm{D}-\mathrm{H} 2 \mathrm{DC}$ & 109.5 \\
\hline $\mathrm{H} 2 \mathrm{DA}-\mathrm{C} 2 \mathrm{D}-\mathrm{H} 2 \mathrm{DC}$ & 109.5 \\
\hline $\mathrm{H} 2 \mathrm{DB}-\mathrm{C} 2 \mathrm{D}-\mathrm{H} 2 \mathrm{DC}$ & 109.5 \\
\hline N1D-C3D-H3DA & 109.5 \\
\hline $\mathrm{N} 1 \mathrm{D}-\mathrm{C} 3 \mathrm{D}-\mathrm{H} 3 \mathrm{DB}$ & 109.5 \\
\hline $\mathrm{H} 3 \mathrm{DA}-\mathrm{C} 3 \mathrm{D}-\mathrm{H} 3 \mathrm{DB}$ & 109.5 \\
\hline $\mathrm{N} 1 \mathrm{D}-\mathrm{C} 3 \mathrm{D}-\mathrm{H} 3 \mathrm{DC}$ & 109.5 \\
\hline $\mathrm{H} 3 \mathrm{DA}-\mathrm{C} 3 \mathrm{D}-\mathrm{H} 3 \mathrm{DC}$ & 109.5 \\
\hline $\mathrm{H} 3 \mathrm{DB}-\mathrm{C} 3 \mathrm{D}-\mathrm{H} 3 \mathrm{DC}$ & 109.5 \\
\hline $\mathrm{C} 1 \mathrm{AD}-\mathrm{O} 1 \mathrm{AD}-\mathrm{Ni2}$ & $122.2(11)$ \\
\hline $\mathrm{O} 1 \mathrm{AD}-\mathrm{C} 1 \mathrm{AD}-\mathrm{N} 1 \mathrm{AD}$ & $128.4(7)$ \\
\hline $\mathrm{O} 1 \mathrm{AD}-\mathrm{C} 1 \mathrm{AD}-\mathrm{H} 1 \mathrm{AD}$ & 115.8 \\
\hline $\mathrm{N} 1 \mathrm{AD}-\mathrm{C} 1 \mathrm{AD}-\mathrm{H} 1 \mathrm{AD}$ & 115.8 \\
\hline $\mathrm{C} 1 \mathrm{AD}-\mathrm{N} 1 \mathrm{AD}-\mathrm{C} 2 \mathrm{AD}$ & $122.6(5)$ \\
\hline $\mathrm{C} 1 \mathrm{AD}-\mathrm{N} 1 \mathrm{AD}-\mathrm{C} 3 \mathrm{AD}$ & $119.2(5)$ \\
\hline $\mathrm{C} 2 \mathrm{AD}-\mathrm{N} 1 \mathrm{AD}-\mathrm{C} 3 \mathrm{AD}$ & $117.0(4)$ \\
\hline $\mathrm{N} 1 \mathrm{AD}-\mathrm{C} 2 \mathrm{AD}-\mathrm{H} 2 \mathrm{DD}$ & 109.5 \\
\hline $\mathrm{N} 1 \mathrm{AD}-\mathrm{C} 2 \mathrm{AD}-\mathrm{H} 2 \mathrm{DE}$ & 109.5 \\
\hline $\mathrm{H} 2 \mathrm{DD}-\mathrm{C} 2 \mathrm{AD}-\mathrm{H} 2 \mathrm{DE}$ & 109.5 \\
\hline $\mathrm{N} 1 \mathrm{AD}-\mathrm{C} 2 \mathrm{AD}-\mathrm{H} 2 \mathrm{DF}$ & 109.5 \\
\hline $\mathrm{H} 2 \mathrm{DD}-\mathrm{C} 2 \mathrm{AD}-\mathrm{H} 2 \mathrm{DF}$ & 109.5 \\
\hline $\mathrm{H} 2 \mathrm{DE}-\mathrm{C} 2 \mathrm{AD}-\mathrm{H} 2 \mathrm{DF}$ & 109.5 \\
\hline N1AD $-\mathrm{C} 3 \mathrm{AD}-\mathrm{H} 3 \mathrm{DD}$ & 109.5 \\
\hline N1AD $-\mathrm{C} 3 \mathrm{AD}-\mathrm{H} 3 \mathrm{DE}$ & 109.5 \\
\hline $\mathrm{H} 3 \mathrm{DD}-\mathrm{C} 3 \mathrm{AD}-\mathrm{H} 3 \mathrm{DE}$ & 109.5 \\
\hline N1AD $-C 3 A D-H 3 D F$ & 109.5 \\
\hline $\mathrm{H} 3 \mathrm{DD}-\mathrm{C} 3 \mathrm{AD}-\mathrm{H} 3 \mathrm{DF}$ & 109.5 \\
\hline $\mathrm{H} 3 \mathrm{DE}-\mathrm{C} 3 \mathrm{AD}-\mathrm{H} 3 \mathrm{DF}$ & 109.5 \\
\hline $\mathrm{C} 1 \mathrm{BD}-\mathrm{O} 1 \mathrm{BD}-\mathrm{Ni} 2$ & $124.0(19)$ \\
\hline $\mathrm{O} 1 \mathrm{BD}-\mathrm{C} 1 \mathrm{BD}-\mathrm{N} 1 \mathrm{BD}$ & $128.4(7)$ \\
\hline $\mathrm{O} 1 \mathrm{BD}-\mathrm{C} 1 \mathrm{BD}-\mathrm{H} 1 \mathrm{BD}$ & 115.8 \\
\hline N1BD $-\mathrm{C} 1 \mathrm{BD}-\mathrm{H} 1 \mathrm{BD}$ & 115.8 \\
\hline $\mathrm{C} 1 \mathrm{BD}-\mathrm{N} 1 \mathrm{BD}-\mathrm{C} 3 \mathrm{BD}$ & $122.5(5)$ \\
\hline
\end{tabular}




\begin{tabular}{|c|c|}
\hline $\mathrm{C} 6 \mathrm{~A}-\mathrm{C} 7 \mathrm{~A}-\mathrm{H} 7 \mathrm{~A}$ & 119.6 \\
\hline $\mathrm{C} 2 \mathrm{~A}-\mathrm{C} 7 \mathrm{~A}-\mathrm{H} 7 \mathrm{~A}$ & 119.6 \\
\hline $\mathrm{O} 4 \mathrm{~A}-\mathrm{C} 8 \mathrm{~A}-\mathrm{O} 3 \mathrm{~A}$ & $126.59(18)$ \\
\hline $\mathrm{O} 4 \mathrm{~A}-\mathrm{C} 8 \mathrm{~A}-\mathrm{C} 5 \mathrm{~A}$ & $116.37(18)$ \\
\hline $\mathrm{O} 3 \mathrm{~A}-\mathrm{C} 8 \mathrm{~A}-\mathrm{C} 5 \mathrm{~A}$ & $117.04(18)$ \\
\hline $\mathrm{C} 5 \mathrm{~A}-\mathrm{C} 4 \mathrm{~A}-\mathrm{C} 3 \mathrm{~A}$ & $120.5(2)$ \\
\hline $\mathrm{C} 5 \mathrm{~A}-\mathrm{C} 4 \mathrm{~A}-\mathrm{H} 4 \mathrm{~A}$ & 119.7 \\
\hline $\mathrm{C} 3 \mathrm{~A}-\mathrm{C} 4 \mathrm{~A}-\mathrm{H} 4 \mathrm{~A}$ & 119.7 \\
\hline $\mathrm{C} 1 \mathrm{~B}-\mathrm{O} 1 \mathrm{~B}-\mathrm{Ni} 2$ & $87.41(13)$ \\
\hline $\mathrm{O} 1 \mathrm{~B}-\mathrm{C} 1 \mathrm{~B}-\mathrm{O} 2 \mathrm{~B}$ & $119.4(2)$ \\
\hline $\mathrm{O} 1 \mathrm{~B}-\mathrm{C} 1 \mathrm{~B}-\mathrm{C} 2 \mathrm{~B}$ & $120.87(19)$ \\
\hline $\mathrm{O} 2 \mathrm{~B}-\mathrm{C} 1 \mathrm{~B}-\mathrm{C} 2 \mathrm{~B}$ & $119.55(19)$ \\
\hline $\mathrm{O} 1 \mathrm{~B}-\mathrm{C} 1 \mathrm{~B}-\mathrm{Ni2}$ & $62.32(12)$ \\
\hline $\mathrm{O} 2 \mathrm{~B}-\mathrm{C} 1 \mathrm{~B}-\mathrm{Ni} 2$ & $57.48(10)$ \\
\hline $\mathrm{C} 2 \mathrm{~B}-\mathrm{C} 1 \mathrm{~B}-\mathrm{Ni2}$ & $169.32(15)$ \\
\hline $\mathrm{Ni} 2-\mathrm{O} 1 \mathrm{~A}-\mathrm{C} 1 \mathrm{~A}-\mathrm{O} 2 \mathrm{~A}$ & $3.0(3)$ \\
\hline $\mathrm{Ni} 2-\mathrm{O} 1 \mathrm{~A}-\mathrm{C} 1 \mathrm{~A}-\mathrm{C} 2 \mathrm{~A}$ & $-177.23(14)$ \\
\hline $\mathrm{O} 1 \mathrm{~A}-\mathrm{C} 1 \mathrm{~A}-\mathrm{O} 2 \mathrm{~A}-\mathrm{Ni1}$ & $16.3(4)$ \\
\hline $\mathrm{C} 2 \mathrm{~A}-\mathrm{C} 1 \mathrm{~A}-\mathrm{O} 2 \mathrm{~A}-\mathrm{Ni1}$ & $-163.41(16)$ \\
\hline $\mathrm{O} 2 \mathrm{~A}-\mathrm{C} 1 \mathrm{~A}-\mathrm{C} 2 \mathrm{~A}-\mathrm{C} 3 \mathrm{~A}$ & $170.1(2)$ \\
\hline $\mathrm{O} 1 \mathrm{~A}-\mathrm{C} 1 \mathrm{~A}-\mathrm{C} 2 \mathrm{~A}-\mathrm{C} 3 \mathrm{~A}$ & $-9.7(3)$ \\
\hline $\mathrm{O} 2 \mathrm{~A}-\mathrm{C} 1 \mathrm{~A}-\mathrm{C} 2 \mathrm{~A}-\mathrm{C} 7 \mathrm{~A}$ & $-8.0(3)$ \\
\hline $\mathrm{O} 1 \mathrm{~A}-\mathrm{C} 1 \mathrm{~A}-\mathrm{C} 2 \mathrm{~A}-\mathrm{C} 7 \mathrm{~A}$ & $172.2(2)$ \\
\hline $\mathrm{C} 7 \mathrm{~A}-\mathrm{C} 2 \mathrm{~A}-\mathrm{C} 3 \mathrm{~A}-\mathrm{C} 4 \mathrm{~A}$ & $1.4(4)$ \\
\hline $\mathrm{C} 1 \mathrm{~A}-\mathrm{C} 2 \mathrm{~A}-\mathrm{C} 3 \mathrm{~A}-\mathrm{C} 4 \mathrm{~A}$ & $-176.8(2)$ \\
\hline $\mathrm{C} 4 \mathrm{~A}-\mathrm{C} 5 \mathrm{~A}-\mathrm{C} 6 \mathrm{~A}-\mathrm{C} 7 \mathrm{~A}$ & $0.1(4)$ \\
\hline $\mathrm{C} 8 \mathrm{~A}-\mathrm{C} 5 \mathrm{~A}-\mathrm{C} 6 \mathrm{~A}-\mathrm{C} 7 \mathrm{~A}$ & $-176.5(3)$ \\
\hline $\mathrm{C} 5 \mathrm{~A}-\mathrm{C} 6 \mathrm{~A}-\mathrm{C} 7 \mathrm{~A}-\mathrm{C} 2 \mathrm{~A}$ & $-0.3(5)$ \\
\hline $\mathrm{C} 3 \mathrm{~A}-\mathrm{C} 2 \mathrm{~A}-\mathrm{C} 7 \mathrm{~A}-\mathrm{C} 6 \mathrm{~A}$ & $-0.4(5)$ \\
\hline $\mathrm{C} 1 \mathrm{~A}-\mathrm{C} 2 \mathrm{~A}-\mathrm{C} 7 \mathrm{~A}-\mathrm{C} 6 \mathrm{~A}$ & $177.8(3)$ \\
\hline $\mathrm{Ni}^{\mathrm{vi}}-\mathrm{O} 4 \mathrm{~A}-\mathrm{C} 8 \mathrm{~A}-\mathrm{O} 3 \mathrm{~A}$ & $-42.2(4)$ \\
\hline $\mathrm{Ni}^{\mathrm{vi}}-\mathrm{O} 4 \mathrm{~A}-\mathrm{C} 8 \mathrm{~A}-\mathrm{C} 5 \mathrm{~A}$ & $137.12(18)$ \\
\hline $\mathrm{Ni} 2^{\mathrm{v}}-\mathrm{O} 3 \mathrm{~A}-\mathrm{C} 8 \mathrm{~A}-\mathrm{O} 4 \mathrm{~A}$ & $24.2(3)$ \\
\hline $\mathrm{Ni} 2^{\mathrm{v}}-\mathrm{O} 3 \mathrm{~A}-\mathrm{C} 8 \mathrm{~A}-\mathrm{C} 5 \mathrm{~A}$ & $-155.13(14)$ \\
\hline $\mathrm{C} 6 \mathrm{~A}-\mathrm{C} 5 \mathrm{~A}-\mathrm{C} 8 \mathrm{~A}-\mathrm{O} 4 \mathrm{~A}$ & $-7.5(3)$ \\
\hline $\mathrm{C} 4 \mathrm{~A}-\mathrm{C} 5 \mathrm{~A}-\mathrm{C} 8 \mathrm{~A}-\mathrm{O} 4 \mathrm{~A}$ & $175.9(2)$ \\
\hline $\mathrm{C} 6 \mathrm{~A}-\mathrm{C} 5 \mathrm{~A}-\mathrm{C} 8 \mathrm{~A}-\mathrm{O} 3 \mathrm{~A}$ & $171.9(2)$ \\
\hline $\mathrm{C} 4 \mathrm{~A}-\mathrm{C} 5 \mathrm{~A}-\mathrm{C} 8 \mathrm{~A}-\mathrm{O} 3 \mathrm{~A}$ & $-4.7(3)$ \\
\hline $\mathrm{C} 6 \mathrm{~A}-\mathrm{C} 5 \mathrm{~A}-\mathrm{C} 4 \mathrm{~A}-\mathrm{C} 3 \mathrm{~A}$ & $0.8(4)$ \\
\hline $\mathrm{C} 8 \mathrm{~A}-\mathrm{C} 5 \mathrm{~A}-\mathrm{C} 4 \mathrm{~A}-\mathrm{C} 3 \mathrm{~A}$ & $177.4(2)$ \\
\hline $\mathrm{C} 2 \mathrm{~A}-\mathrm{C} 3 \mathrm{~A}-\mathrm{C} 4 \mathrm{~A}-\mathrm{C} 5 \mathrm{~A}$ & $-1.6(4)$ \\
\hline $\mathrm{Ni2}-\mathrm{O} 1 \mathrm{~B}-\mathrm{C} 1 \mathrm{~B}-\mathrm{O} 2 \mathrm{~B}$ & $6.94(19)$ \\
\hline
\end{tabular}

$\begin{array}{ll}\text { C1BD-N1BD-C2BD } & 119.2(5) \\ \text { C3BD-N1BD-C2BD } & 117.0(4) \\ \text { N1BD-C2BD-H2DG } & 109.5 \\ \text { N1BD-C2BD-H2DH } & 109.5 \\ \text { H2DG-C2BD-H2DH } & 109.5 \\ \text { N1BD-C2BD-H2DI } & 109.5 \\ \text { H2DG-C2BD-H2DI } & 109.5 \\ \text { H2DH-C2BD-H2DI } & 109.5 \\ \text { N1BD-C3BD-H3DG } & 109.5 \\ \text { N1BD-C3BD-H3DH } & 109.5 \\ \text { H3DG-C3BD-H3DH } & 109.5 \\ \text { N1BD-C3BD-H3DI } & 109.5 \\ \text { H3DG-C3BD-H3DI } & 109.5 \\ \text { H3DH-C3BD-H3DI } & 109.5\end{array}$

$\mathrm{Ni2}-\mathrm{O} 1 \mathrm{~B}-\mathrm{C} 1 \mathrm{~B}-\mathrm{C} 2 \mathrm{~B} \quad-168.31$ (18)

$\mathrm{O} 1 \mathrm{~B}-\mathrm{C} 1 \mathrm{~B}-\mathrm{O} 2 \mathrm{~B}-\mathrm{Ni2} \quad-7.3(2)$

$\mathrm{C} 2 \mathrm{~B}-\mathrm{C} 1 \mathrm{~B}-\mathrm{O} 2 \mathrm{~B}-\mathrm{Ni2} \quad 168.02(17)$

$\mathrm{O} 1 \mathrm{~B}-\mathrm{C} 1 \mathrm{~B}-\mathrm{O} 2 \mathrm{~B}-\mathrm{Ni1} \quad 113.0(2)$

$\mathrm{C} 2 \mathrm{~B}-\mathrm{C} 1 \mathrm{~B}-\mathrm{O} 2 \mathrm{~B}-\mathrm{Ni1} \quad-71.7(2)$

$\mathrm{Ni} 2-\mathrm{C} 1 \mathrm{~B}-\mathrm{O} 2 \mathrm{~B}-\mathrm{Ni1} \quad 120.27(16)$

$\mathrm{C} 4 \mathrm{~B}-\mathrm{C} 3 \mathrm{~B}-\mathrm{C} 2 \mathrm{~B}-\mathrm{C} 4 \mathrm{~B}^{\mathrm{iv}} \quad 0.5(4)$

$\mathrm{C} 4 \mathrm{~B}-\mathrm{C} 3 \mathrm{~B}-\mathrm{C} 2 \mathrm{~B}-\mathrm{C} 1 \mathrm{~B} \quad-177.2(2)$

$\mathrm{O} 1 \mathrm{~B}-\mathrm{C} 1 \mathrm{~B}-\mathrm{C} 2 \mathrm{~B}-\mathrm{C} 3 \mathrm{~B} \quad 157.8(2)$

$\mathrm{O} 2 \mathrm{~B}-\mathrm{C} 1 \mathrm{~B}-\mathrm{C} 2 \mathrm{~B}-\mathrm{C} 3 \mathrm{~B} \quad-17.4(3)$

$\mathrm{Ni} 2-\mathrm{C} 1 \mathrm{~B}-\mathrm{C} 2 \mathrm{~B}-\mathrm{C} 3 \mathrm{~B} \quad 53.4(9)$

$\mathrm{O} 1 \mathrm{~B}-\mathrm{C} 1 \mathrm{~B}-\mathrm{C} 2 \mathrm{~B}-\mathrm{C} 4 \mathrm{~B}^{\mathrm{iv}} \quad-19.9(3)$

$\mathrm{O} 2 \mathrm{~B}-\mathrm{C} 1 \mathrm{~B}-\mathrm{C} 2 \mathrm{~B}-\mathrm{C} 4 \mathrm{~B}^{\mathrm{iv}} \quad 164.9$ (2)

$\mathrm{Ni} 2-\mathrm{C} 1 \mathrm{~B}-\mathrm{C} 2 \mathrm{~B}-\mathrm{C}^{2} \mathrm{~B}^{\mathrm{iv}} \quad-124.3(8)$

$\mathrm{C} 2 \mathrm{~B}-\mathrm{C} 3 \mathrm{~B}-\mathrm{C} 4 \mathrm{~B}-\mathrm{C}_{2} \mathrm{~B}^{\mathrm{iv}} \quad-0.5(4)$

$\mathrm{Ni} 2-\mathrm{O} 1 \mathrm{C}-\mathrm{C} 1 \mathrm{C}-\mathrm{N} 1 \mathrm{C} \quad-159.3(2)$

$\mathrm{O} 1 \mathrm{C}-\mathrm{C} 1 \mathrm{C}-\mathrm{N} 1 \mathrm{C}-\mathrm{C} 3 \mathrm{C} \quad 1.8(6)$

$\mathrm{O} 1 \mathrm{C}-\mathrm{C} 1 \mathrm{C}-\mathrm{N} 1 \mathrm{C}-\mathrm{C} 2 \mathrm{C} \quad-177.7(3)$

$\mathrm{Ni2}-\mathrm{O} 1 \mathrm{D}-\mathrm{C} 1 \mathrm{D}-\mathrm{N} 1 \mathrm{D} \quad 153.1(8)$

$\mathrm{O} 1 \mathrm{D}-\mathrm{C} 1 \mathrm{D}-\mathrm{N} 1 \mathrm{D}-\mathrm{C} 2 \mathrm{D} \quad 172.9(11)$

$\mathrm{O} 1 \mathrm{D}-\mathrm{C} 1 \mathrm{D}-\mathrm{N} 1 \mathrm{D}-\mathrm{C} 3 \mathrm{D} \quad-2.2(15)$

$\mathrm{Ni2}-\mathrm{O} 1 \mathrm{AD}-\mathrm{C} 1 \mathrm{AD}-\mathrm{N} 1 \mathrm{AD} \quad-165.3(13)$

$\mathrm{O} 1 \mathrm{AD}-\mathrm{C} 1 \mathrm{AD}-\mathrm{N} 1 \mathrm{AD}-\mathrm{C} 2 \mathrm{AD}-177(3)$

$\mathrm{O} 1 \mathrm{AD}-\mathrm{C} 1 \mathrm{AD}-\mathrm{N} 1 \mathrm{AD}-\mathrm{C} 3 \mathrm{AD}-10(3)$

$\mathrm{Ni2}-\mathrm{O} 1 \mathrm{BD}-\mathrm{C} 1 \mathrm{BD}-\mathrm{N} 1 \mathrm{BD} \quad-154.2(15)$

O1BD-C1BD-N1BD-C3BD $22(3)$

O1BD-C1BD-N1BD-C2BD $-172(3)$

Symmetry codes: (i) $-x+1,-y+1,-z+1$; (ii) $x+1 / 2,-y+3 / 2, z+1 / 2$; (iii) $-x+1 / 2, y-1 / 2,-z+1 / 2$; (iv) $-x+1,-y,-z+1$; (v) $x-1 / 2,-y+3 / 2, z-1 / 2$; (vi) $-x+1 / 2$, $y+1 / 2,-z+1 / 2$. 
supporting information

Hydrogen-bond geometry $\left(\AA,{ }^{\circ}\right)$

\begin{tabular}{lllll}
\hline$D-\mathrm{H} \cdots A$ & $D-\mathrm{H}$ & $\mathrm{H} \cdots A$ & $D \cdots A$ & $D-\mathrm{H} \cdots A$ \\
\hline $\mathrm{C} 1 C-\mathrm{H} 1 C \cdots \mathrm{O} 1 A$ & 0.93 & 2.33 & $2.893(3)$ & 119 \\
$\mathrm{C} 2 C-\mathrm{H} 2 C B \cdots \mathrm{O} 1 B^{\text {vii }}$ & 0.96 & 2.56 & $3.367(5)$ & 143 \\
$\mathrm{C} 1 A D-\mathrm{H} 1 A D^{\cdots} \mathrm{O} 2 B$ & 0.93 & 2.49 & $3.013(7)$ & 116 \\
$\mathrm{C} 2 A D-\mathrm{H} 2 D E \cdots \mathrm{O} 1 B^{\text {viii }}$ & 0.96 & 2.39 & $3.089(8)$ & 130 \\
$\mathrm{C} 2 B D-\mathrm{H} 2 D G \cdots \mathrm{O} 1 B^{\text {viii }}$ & 0.96 & 2.39 & $2.981(10)$ & 119
\end{tabular}

Symmetry codes: (vii) $-x+3 / 2, y+1 / 2,-z+1 / 2$; (viii) $x+1 / 2,-y+1 / 2, z+1 / 2$. 